\title{
Particle-in-cell simulation of a mildly relativistic collision of an electron-ion plasma carrying a quasi-parallel magnetic field ${ }^{\star}$
}

\section{Electron acceleration and magnetic field amplification at supernova shocks}

\author{
M. E. Dieckmann ${ }^{1}$, G. C. Murphy ${ }^{2}$, A. Meli $^{3}$, and L. O. C. Drury ${ }^{2}$ \\ 1 Department of Science and Technology, Linkoping University, 60174 Norrkoping, Sweden \\ e-mail: Mark.E.Dieckmann@itn.liu.se \\ 2 Dublin Institute for Advanced Studies, 31 Fitzwilliam Place, Dublin 2, Ireland \\ 3 Center for Astroparticle Physics, Friedrich-Alexander-University Erlangen-Nuremberg, 91058 Erlangen, Germany
}

Received 5 June 2009 / Accepted 14 September 2009

\begin{abstract}
Context. Plasma processes close to supernova remnant shocks result in the amplification of magnetic fields and in the acceleration of electrons, injecting them into the diffusive acceleration mechanism.

Aims. The acceleration of electrons and the magnetic field amplification by the collision of two plasma clouds, each consisting of electrons and ions, at a speed of $0.5 \mathrm{c}$ is investigated. A quasi-parallel guiding magnetic field, a cloud density ratio of 10 and a plasma temperature of $25 \mathrm{keV}$ are considered.

Methods. A relativistic and electromagnetic particle-in-cell simulation models the plasma in two spatial dimensions employing an ion-to-electron mass ratio of 400.

Results. A quasi-planar shock forms at the front of the dense plasma cloud. It is mediated by a circularly left-hand polarized electromagnetic wave with an electric field component along the guiding magnetic field. Its propagation direction is close to that of the guiding field and orthogonal to the collision boundary. It has a frequency too low to be determined during the simulation time and a wavelength that equals several times the ion inertial length. These properties would be indicative of a dispersive Alfvén wave close to the ion cyclotron resonance frequency of the left-handed mode, known as the ion whistler, provided that the frequency is appropriate. However, it moves with the super-alfvénic plasma collision speed, suggesting that it is an Alfvén precursor or a nonlinear MHD wave such as a Short Large-Amplitude Magnetic Structure (SLAMS). The growth of the magnetic amplitude of this wave to values well in excess of those of the quasi-parallel guiding field and of the filamentation modes results in a quasi-perpendicular shock. We present evidence for the instability of this mode to a four wave interaction. The waves developing upstream of the dense cloud give rise to electron acceleration ahead of the collision boundary. Energy equipartition between the ions and the electrons is established at the shock and the electrons are accelerated to relativistic speeds.

Conclusions. The magnetic fields in the foreshock of supernova remnant shocks can be amplified substantially and electrons can be injected into the diffusive acceleration, if strongly magnetised plasma subshells are present in the foreshock, with velocities an order of magnitude faster than the main shell.
\end{abstract}

Key words. plasmas - acceleration of particles - magnetic fields - shock waves - methods: numerical - ISM: supernova remnants

\section{Introduction}

Supernova remnants (SNRs) emanate energetic electromagnetic radiation, which demonstrates the acceleration of electrons to ultrarelativistic speeds (Tanimori et al. 1998; Uchiyama et al. 2007) and the generation or amplification of magnetic fields (Ellison \& Vladimirov 2008; Volk et al. 2005). The likely origin of the accelerated electrons and of the strong magnetic fields is the SNR shock (Marcowith et al. 2006; Pelletier et al. 2006).

The nonrelativistic expansion speed of the main SNR blast shell (Kulkarni et al. 1998; Fransson et al. 2002) and the weak magnetic field of the ambient medium (Volk et al. 2005), into which this shell is expanding, are obstacles to the magnetic field amplification by plasma instabilities and to the electron acceleration out of the thermal plasma distribution to moderately relativistic energies. Such an acceleration is needed for their injection (Cargill \& Papadopoulos 1988; Kirk \& Dendy 2001;

$\star 5$ movies are only available in electronic form at http: //www . aanda. org
Kuramitsu \& Krasnoselskikh 2005a,b) into the diffusive shock acceleration process (See Drury 1983) so that they can cross the shock transition layer repeatedly. Electrostatic instabilities dominate for nonrelativistic flows in unmagnetized plasmas (Bret et al. 2008; Bret 2009) and they can neither accelerate the electrons to highly relativistic speeds (Sircombe et al. 2006) nor amplify the magnetic fields.

The electrons could be accelerated by plasma based charged particle accelerators (Bingham et al. 2004), by electron surfing acceleration (Katsouleas \& Dawson 1983; Ohira \& Takahara 2007; Dieckmann et al. 2008a), double layers (Raadu \& Rasmussen 1988; Dieckmann \& Bret 2009) or by processes that exploit a velocity shear in the plasma outflow (Rieger \& Duffy 2006). It has, however, not yet been demonstrated with multidimensional and self-consistent simulations that these mechanisms can indeed achieve the required electron acceleration and magnetic field amplification. The non-relativistic shocks, which are found between the main SNR blast shell and the ambient medium, can also probably not transfer significant energy from 
the ions to the electrons and accelerate the latter to relativistic speeds (Hoshino \& Shimada 2002; Lembege et al. 2004; Scholer \& Matsukiyo 2004; Chapman et al. 2005; Sorasio et al. 2006; Amano \& Hoshino 2007; Umeda et al. 2009).

A viable acceleration mechanism may develop ahead of the main SNR shock, if we find subshells that outrun the main blast shell. These subshells may move faster than the typical peak speed of $0.2 \mathrm{c}$ of the main shell. An expansion speed as high as $0.9 \mathrm{c}$ may have been observed for a subshell ejected by the supernova SN1998bw (Kulkarni et al. 1998). Most supernovae are less violent and their subshells are probably slower. The density of the subshell plasma is well below that of the main blast shell and its dynamics will be influenced to a larger extent by the upstream magnetic field than the dynamics of the latter. This is true in particular, if the upstream magnetic field has been preamplified by the cosmic rays (Winske \& Leroy 1984; Bell 2004; Reville et al. 2007; Niemiec et al. 2008; Riquelme \& Spitkovsky 2009). A fast magnetized shock would form in the foreshock of the main SNR shock that can result in a stronger magnetic field amplification and electron acceleration.

We examine with a particle-in-cell (PIC) simulation the formation of a shock in a plasma, in which a strong guiding magnetic field is quasi-parallel to the plasma expansion direction. Whistler waves, which become Alfvén waves at low frequencies, occuring at such shocks can be efficient electron accelerators (Levinson 1992; Kuramitsu \& Krasnoselskikh 2005a,b; Miteva \& Mann 2007; McClements \& Fletcher 2009). Whistlers and Alfvén waves are circulary polarized if they propagate parallel to the guiding magnetic field. We briefly summarize their properties and focus on the low-frequency modes with a left-hand polarization. These modes are qualitatively similar to the quasiparallel propagating ones we consider here. A more thorough description of the dispersion relation of two-fluid waves and the shift of the resonance frequencies for quasi-parallel propagation can be found elsewhere (Treumann \& Baumjohann 1997).

The dominant waves, which we will observe, grow in a plasma with an electron gyrofrequency that exceeds the plasma frequency. As we increase the frequency in such a strongly magnetized plasma towards the ion cyclotron frequency, the Alfvén waves with a left-hand circular polarization become dispersive. These waves resonate with the ions and their frequencies remain below the ion cyclotron frequency. Alfvén modes with a lefthand circular polarization just below the ion cyclotron frequency are called ion whistlers. Whistlers are predominantly electromagnetic for small propagation angles relative to the guiding magnetic field, as it has been discussed for high-frequency ones by Tokar \& Gary (1985), and if they have low wavenumbers. Ion whistlers or dispersive Alfvén waves develop a field-aligned electric field component close to the resonance frequency, by which they can interact nonlinearly with the plasma particles and accelerate them (McClements \& Fletcher 2009). Any wave growth will furthermore result in an increasing energy density of the magnetic field.

Alfvén waves and other magnetohydrodynamic (MHD) waves can grow to amplitudes, at which they start to interact nonlinearly with the plasma (Stasiewicz \& Ekeberg 2008). Short Large Amplitude Magnetic Field Structures (SLAMS) are nonlinear MHD waves occuring at quasi-parallel shocks and may be relevant for our simulation. The SLAMS can be efficient electron accelerators (Mann \& Classen 1997). Their magnetic amplitude reaches several times that of the background field and they can propagate with a super-Alfvénic speed, because they convect with the ions (Mann et al. 1994; Behlke et al. 2003). SLAMS have also been observed in simulations
(Scholer et al. 2003). The acceleration of electrons by substructures of quasi-parallel shocks, which may be SLAMS, has been observed in the solar corona (Mann et al. 1998).

The absence of self-consistent kinetic models of oblique shocks implies that they can currently be studied only numerically with particle-in-cell (PIC) (Dawson 1983; Eastwood 1991) or with Vlasov simulations (Arber \& Vann 2002; Sircombe et al. 2006). The pioneering PIC simulations of plasma slabs, which collide with a speed of $0.9 c$ and in the presence of an oblique magnetic field (Bessho \& Ohsawa 1999; Zindo et al. 2005), have evidenced the formation of a shock that accelerated the electrons to ultrarelativistic speeds and amplified the magnetic field. A more recent PIC simulation study (Dieckmann et al. 2008b) has shown, that the shock formation is triggered by an energetic electromagnetic structure (EES). The simulation could demonstrate that an approximate equipartition of the ion, electron and magnetic energy densities is established. However, these simulations could only resolve one spatial dimension due to computer constraints, which is not necessarily realistic for mildly relativistic collision speeds.

We perform here a case study with initial conditions, which are similar to those employed by Dieckmann et al. (2008b). We reduce the collision speed to $0.5 c$ and lower the temperature. The plasma cloud representing the subshell is ten times denser than the plasma cloud that represents the ambient plasma (interstellar medium), into which the shell expands. A guiding magnetic field is quasi-parallel to the plasma flow velocity vector and it results in an electron gyrofrequency that equals the electron plasma frequency of the dense cloud. These bulk plasma parameters have been selected with the intention to enforce a planar shock front (Hededal \& Nishikawa 2005; Bret et al. 2006; Dieckmann et al. 2008b), by which we can model the shock in one- or two-dimensions in space. The reduced ion-to-electron mass ratio we use allows us to model this collision in form of a 2(1/2)D particle-in-cell (PIC) simulation, which resolves two spatial and three momentum dimensions.

Our results are summarized as follows: we find higherdimensional structures (density filaments), which initially form at the front of the tenuous plasma cloud and expand in time. The front of the dense cloud, which turns out to be the most relevant structure, remains planar and its filamentation is delayed but not suppressed by the guiding magnetic field and the high temperature. An EES grows ahead of the dense plasma cloud before it has become filamentary and the magnetic amplitude of the EES reaches a value several times the one of the initial guiding magnetic field. The EES is pushed by the dense cloud and its phase speed in the rest frame of the tenuous cloud is comparable to the cloud collision speed or twice the Alfvén speed in the tenuous cloud. The front of the EES expands at an even higher speed. Its high speed and amplitude may imply that the EES is a SLAMS. The front of the dense cloud and, consequently, the EES are slowed down by the electromagnetic wave-particle interaction. It is thus not possible to define a rest frame moving with a constant speed, in which the EES is stationary. This would be necessary to measure the frequency of the EES accurately. However, the amplitude distribution of the EES suggests that its oscillation frequency is below the ion cyclotron frequency. Electromagnetic waves are destabilized by the EES ahead of the cloud overlap layer through what we think is a four-wave interaction (Goldstein 1978). The electrons are accelerated in the combined wave fields of these waves and in the forming shock to highly relativistic speeds. The simulation shows though that the strongest electron acceleration occurs at the position, where the shock-reflected ion beam is forming. The plasma collision 


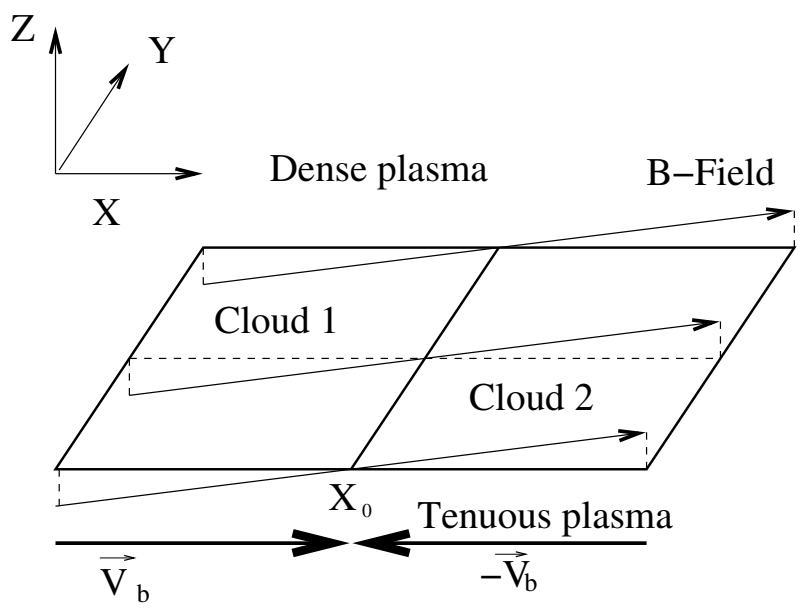

Fig. 1. Initial conditions. Two clouds are modelled. Each cloud occupies one half space and their mean velocity vectors $\boldsymbol{v}_{b}$ and $-\boldsymbol{v}_{b}$ point along the x-direction. The background magnetic field $\boldsymbol{B}_{0} \|(1,0,0.1)$. A convection electric field points along $\boldsymbol{y}$.

results in a substantial electron acceleration and also in an amplification of the magnetic energy density by one order of magnitude within the EES and the forming shock. Both values are probably limited by the reduced ion mass of 400 electron masses, which we must employ. Radiative processes, which are not resolved by the PIC code, will at this stage start to influence the shock evolution (Schlickeiser \& Lerche 2007, 2008) and we stop the simulation.

This paper is structured as follows. Section 2 discusses the particle-in-cell simulation method and the initial conditions. Section 3 presents our results, which are discussed in more detail in Sect. 4.

\section{Initial conditions and simulation method}

We model the collision of two plasma clouds. Each cloud consists of co-moving and equally dense electron and ion species. This system is sketched in Fig. 1. The dense species represents in this model a fast subshell of the SNR blast shell and the tenuous species the ambient medium.

The number densities of the electrons with the charge $-e$ and mass $m_{\mathrm{e}}$ of the dense cloud 1 and of the tenuous cloud 2 are $n_{\mathrm{e} 1}$ and $n_{\mathrm{e} 2}=n_{\mathrm{e} 1} / 10$. The number densities of the ions with the charge and mass e and $m_{i}$ of cloud 1 and 2 are $n_{i 1}=n_{\mathrm{e} 1}$ and $n_{i 2}=n_{\mathrm{e} 2}$. The cloud 1 occupies the half space $x<x_{0}$ and the cloud 2 occupies $x>x_{0}$. Both clouds collide at the initial contact boundary, which we set to $x_{0}=0$. All four species are initialized in their respective rest frame with a relativistic Maxwellian distribution that has the temperature $T=25 \mathrm{keV}$. This value exceeds by far the temperature of the ambient medium (ISM) into which the blast shell expands, which is typically less than $1 \mathrm{eV}$. The upstream temperature may, however, be increased by the interaction of the ISM with the cosmic ray precursor of the main blast shell. The temperature $T$ is also higher than that downstream of the SNR shock, which is usually a few keV. The purpose of selecting this high temperature of the simulation plasma is to improve the numerical efficiency. It maximizes the grid cell size, which must be comparable to the plasma Debye length, and the time step that is connected to the cell size, while it ensures that no highly relativistic particles are present to start with.

We set $v_{b}=0.268 \mathrm{c}$ and the cloud collision speed $v_{c}=$ $2 v_{b} /\left(1+v_{b}^{2} / c^{2}\right)$ is $v_{c}=c / 2$. The thermal speed $v_{\mathrm{e} 1}=\left(k_{B} T / m_{\mathrm{e}}\right)^{1 / 2}$ and $v_{\mathrm{e} 2}=v_{\mathrm{e} 1}$ of the electrons of the clouds 1 and 2 is $v_{\mathrm{e} 1} \approx$ $0.825 v_{b}$. The jump of the electron's mean velocity at $x=x_{0}$ is thus comparable to the thermal speed and the discontinuity in the electron phase space distribution is not strong. The thermal speeds $v_{i 1}=v_{i 2}$ of both ion species are $v_{i 1}=\left(m_{\mathrm{e}} / m_{i}\right)^{0.5} v_{\mathrm{e} 1}$. The guiding magnetic field $\boldsymbol{B}_{0}=B_{x, 0}(1,0,0.1)$ with $e B_{x, 0} / m_{\mathrm{e}}=\Omega_{\mathrm{e} 1}$, where $\Omega_{\mathrm{e} 1}=\left(n_{\mathrm{e} 1} e^{2} / m_{\mathrm{e}} \epsilon_{0}\right)^{1 / 2}$ is the electron plasma frequency of cloud 1. The convection electric field with the modulus $E_{c}=$ $v_{b} B_{z, 0}$ with $B_{z, 0}=B_{x, 0} / 10$ points along $\boldsymbol{y}$ and changes its sign at $x=x_{0}$. The Alfvén speed in cloud 2 is $V_{\mathrm{A}} \approx 0.23$ in units of $c$ and the collision has an initial Alfvén Mach number $\approx 2$. The $\beta=2 \mu_{0}\left(n_{\mathrm{e} 1}+n_{i 1}\right) k_{B} T / B_{0}^{2}=0.2$, where $T$ is given in units of Kelvin, and the plasma is strongly magnetized. However, in the box frame of reference, the average kinetic energy density of the plasma exceeds the magnetic energy density by the factor 8.4 and the kinetic energy thus still dominates.

Typical particle number densities of the ambient interstellar medium, into which the plasma subshell would expand, are $0.1 \mathrm{~cm}^{-3}<n_{0}<1.0 \mathrm{~cm}^{-3}$ (Acero et al. 2007; Ellison \& Vladimirov 2008). This ambient medium would correspond to the cloud 2. These densities are not always known, they change as a function of space and are not the same for all SNRs. We will thus use normalized units that permit us to scale the simulation results to any $n_{0}$.

Variables in physical units are denoted by the subscript $p$. The time and space are scaled to $t=\Omega_{\mathrm{e} 1} t_{p}$ and $\boldsymbol{x}=\lambda_{\mathrm{s}}^{-1} \boldsymbol{x}_{p}$, where $\lambda_{\mathrm{s}}=c / \Omega_{\mathrm{e} 1}$ is the electron skin depth of cloud 1 . The ion skin depth equals $20 \lambda_{\mathrm{s}}$ for the considered ion-to-electron mass ratio. The electric and magnetic fields are scaled to $\boldsymbol{E}_{p}\left(\lambda_{\mathrm{s}} \boldsymbol{x}\right)=$ $\Omega_{\mathrm{e} 1} c m_{\mathrm{e}} e^{-1} \boldsymbol{E}(\boldsymbol{x})$ and $\boldsymbol{B}_{p}\left(\lambda_{\mathrm{s}} \boldsymbol{x}\right)=\Omega_{\mathrm{e} 1} m_{\mathrm{e}} e^{-1} \boldsymbol{B}(\boldsymbol{x})$. The charge, the particle number density and the current are normalized as $\rho_{p}\left(\lambda_{\mathrm{s}} \boldsymbol{x}\right)=e n_{\mathrm{e} 1} \rho(\boldsymbol{x}), n_{p}\left(\lambda_{\mathrm{s}} \boldsymbol{x}\right)=n_{\mathrm{e} 1} n(\boldsymbol{x})$ and $\boldsymbol{J}_{p}\left(\lambda_{\mathrm{s}} \boldsymbol{x}\right)=$ $e n_{\mathrm{e} 1} c \boldsymbol{J}(\boldsymbol{x})$. The normalized Maxwell's equations are

$$
\begin{aligned}
& \nabla \times \boldsymbol{E}=-\frac{\partial \boldsymbol{B}}{\partial t}, \nabla \cdot \boldsymbol{B}=0, \\
& \nabla \times \boldsymbol{B}=\frac{\partial \boldsymbol{E}}{\partial t}+\boldsymbol{J}, \nabla \cdot \boldsymbol{E}=\rho .
\end{aligned}
$$

A PIC code solves the Klimontovich-Dupree equations (Dupree 1963) that are derived from the Vlasov equation with the help of the method of characteristics. The equations of motion

$\frac{\mathrm{d} \boldsymbol{p}_{j}}{\mathrm{~d} t}=q_{r}\left(\boldsymbol{E}\left(\boldsymbol{x}_{j}\right)+\boldsymbol{v}_{j} \times \boldsymbol{B}\left(\boldsymbol{x}_{j}\right)\right)$

$\boldsymbol{p}_{j}=m_{r} \gamma\left(\boldsymbol{v}_{j}\right) \boldsymbol{v}_{j}, \frac{\mathrm{d} \boldsymbol{x}_{j}}{\mathrm{~d} t}=\boldsymbol{v}_{j}$

are solved for an ensemble of computational particles (CPs), where the subscript $j$ denotes one $\mathrm{CP}$ of the species $r$. The charge $q_{r}$ and mass $m_{r}$ of the CP are given in units of e and $m_{\mathrm{e}}$ and the momentum is normalized to $m_{\mathrm{e}} c$. The macroscopic current $\boldsymbol{J}$ is obtained from interpolating the current contributions of each CP to the grid and from the summation of these interpolating currents over all particles. The macroscopic $\boldsymbol{J}$ is used to evolve $\boldsymbol{E}$ and $\boldsymbol{B}$ one step in time. The momentum $\boldsymbol{p}_{j}$ of each $\mathrm{CP}$ with index $j$ is then updated with these new electromagnetic fields, which are interpolated to the position $\boldsymbol{x}_{j}$ of the respective CP. Interpolation schemes are discussed by Dawson (1983) and the one our code uses by Eastwood (1991).

Our code is relativistic and electromagnetic and we use periodic boundary conditions in all directions. The simulation represents the $x, y$ plane. The simulation box length is $L_{x}=5330$ along $x$, which is resolved by $1.8 \times 10^{4}$ grid cells. The length 


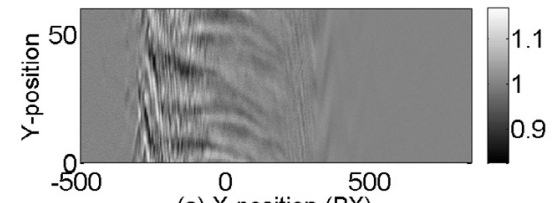

(a) X-position (BX)

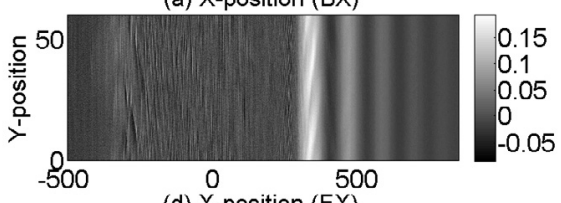

(d) X-position (EX)

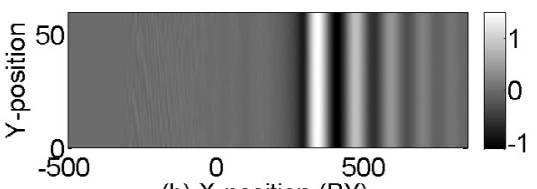

(b) X-position (BY)

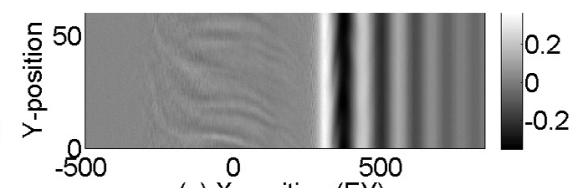

(e) X-position (EY)

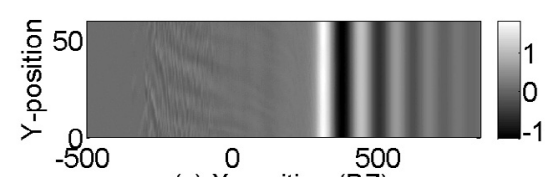

(c) X-position (BZ)

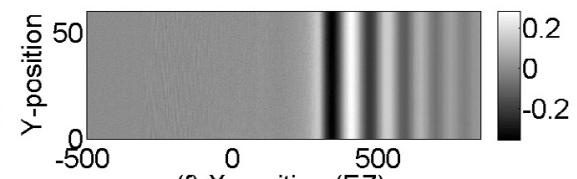

(f) X-position (EZ)

Fig. 2. Electromagnetic field amplitudes in the simulation at the time $T_{1}$. The upper row shows the magnetic field components $B_{x}(x, y)$ a), $B_{y}(x, y)$ b) and $B_{z}(x, y)$ c). The lower row displays the $\left.E_{x}(x, y) \mathbf{d}\right), E_{y}(x, y)$ e) and $\left.E_{z}(x, y) \mathbf{f}\right)$.

$L_{y}=60$ along $y$ is resolved by 200 grid cells. Cloud 1 occupies the x-interval $-L_{x} / 2<x<0$, while cloud 2 occupies $0<x<L_{x} / 2$. No new particles are introduced at the boundaries. The clouds convect away from the initial contact boundary at $x=0$ with the speed modulus $v_{b}$ and the simulation is stopped before the rear boundaries of the clouds enter the area of interest.

Each plasma species is represented by $160 \mathrm{CPs}$ per cell. The density difference of both clouds thus implies a lower weight for the CPs of cloud 2. We employ a mass ratio $m_{i} / m_{\mathrm{e}}=400$ and let the simulation run for the duration $T_{\text {sim }}=3500$. If no strong instabilities develop, the particles could move on average a distance $v_{b} T_{\text {sim }}=950$.

\section{Simulation results}

In what follows, we analyse the data at two times. The field and particle distribution is considered at the early time $T_{1}=1150$. We examine, which conditions eventually result in the growth of the shock. The shock is forming at the later time $T_{\text {sim }}$ and we determine the plasma state that is reached by the release of the ion kinetic energy.

\subsection{Early simulation time}

Figure 2 displays the fields at the time $T_{1}$. The spatial interval $-300<x<300$, in which the clouds overlap by $v_{b} T_{1} \approx 300$, shows filamentary structures in $B_{x}, B_{z}$ and $E_{y}$. We discuss these first. The filamentation instability separates the currents due to both interpenetrating clouds and a current system develops in the simulation plane. It drives the $B_{z}$, which gives rise to the $E_{y}$ through linear (Tzoufras et al. 2006) and nonlinear processes (Dieckmann et al. 2009). The $B_{z}$ is modulated along $y$ with a wavelength between $5 \lambda_{\mathrm{s}}$ and $20 \lambda_{\mathrm{s}}$, the latter being equal to the ion skin depth. At least the largest structures of $B_{z}$ seem to be tied to ion filaments.

Initially the convection electric field along $y$ ensures that the magnetic field is co-moving with the plasma. The gyrocenters of the plasma particles do not move relative to $\boldsymbol{B}_{0}$ and no net current is present. Once the plasma clouds overlap, there will be a net particle motion relative to $\boldsymbol{B}_{0}$ and the plasma particles can be deflected into the $y, z$-plane. The derivative along $z$ vanishes in our 2D PIC simulation. The $B_{x}$ can only be generated through $\partial_{x} B_{y}-\partial_{y} B_{x}=\partial_{t} E_{z}+J_{z}$, which causes structure formation in $B_{x}$ and $B_{y}$. Figure 2a reveals that the filamentation of $B_{x}$ due to such currents is strongest close to the front of cloud 2, that is for $x<0$. The plots of $B_{y}$ and $E_{z}$ do not yet show a clear filamentation but one has to keep in mind that the apparent relative weakness of the modulations of $B_{y}$ compared to those of $B_{x}$ is influenced by

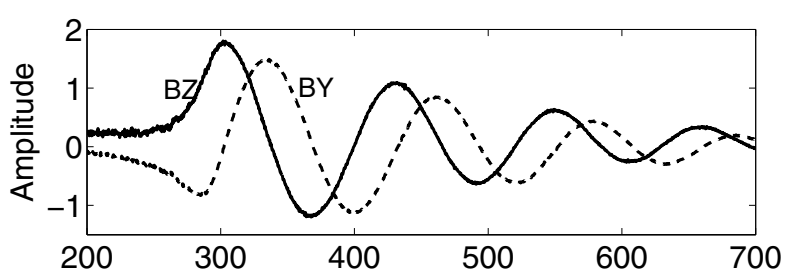

(a) X-position: BY,BZ

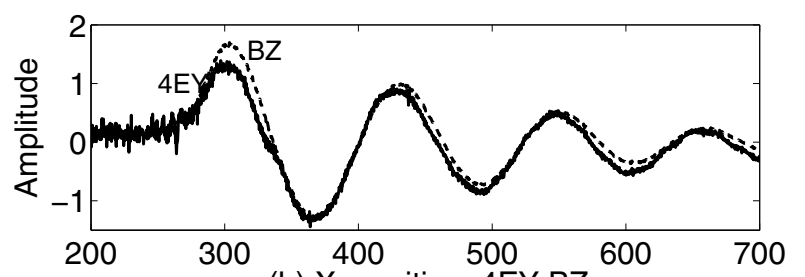

(b) X-position: 4EY,BZ

Fig. 3. Comparison of the planar electromagnetic fields along $x$ for $y=$ 0 . a) compares $c B_{z}$ with $c B_{y}$ in units of $c B_{x, 0}$, while b) compares $c B_{z}$ with $4 E_{y}$ in the same normalization.

the different colour scales. The fluctuations of $E_{x}$ in the interval $-300<x<300$ have no visible counterpart in $B_{y}$ and $B_{z}$. The polarization of the electric field fluctuations along $\boldsymbol{v}_{b}$ suggests a charge density wave, but their correlation with the small-scale fluctuations of $B_{x}$ for $-300<x<-100$ demonstrates that they are not purely electrostatic.

The dominant fields of the energetic electromagnetic structure (EES) are observed in Fig. 2 ahead of the dense cloud 1 for $x>300$. The EES is planar. The field amplitudes along $y, z$ are practically constant along $y$. The $B_{y}$ and $B_{z}$ in Figs. $2 \mathrm{~b}, \mathrm{c}$ reach peak amplitudes exceeding that of the guiding magnetic field in this interval. The $E_{x}$ and $B_{x}$ at $x \approx 300$ show an oblique modulation.

We exploit the planarity and constancy as a function of $y$ of the amplitudes of $B_{y}, B_{z}$ and $E_{y}$ for $x>250$, where we find the EES. Figure 3 plots these amplitudes as a function of $x$ for $y=0$. The considered field components have their peak values at the front of cloud 1 located at $x \approx 300$. The amplitudes decrease exponentially for $x<300$ and $B_{y}$ and $B_{z}$ reveal a phase shift of $180^{\circ}$. The amplitudes of $B_{y}, B_{z}$ and $E_{y}$ decrease also for increasing $x>300$, although more slowly and the fields oscillate. The phases of $B_{y}$ and $B_{z}$ are shifted by $\approx 90^{\circ}$ in this interval and the EES is predominantly circularly polarized. The electron gyrofrequency is comparable to the plasma frequency for $x<300$ and significantly larger for $x>300$. The jump in the ratio of the characteristic resonance frequencies at $x \approx 300$ will alter the wave dispersive properties of the plasma, explaining the 


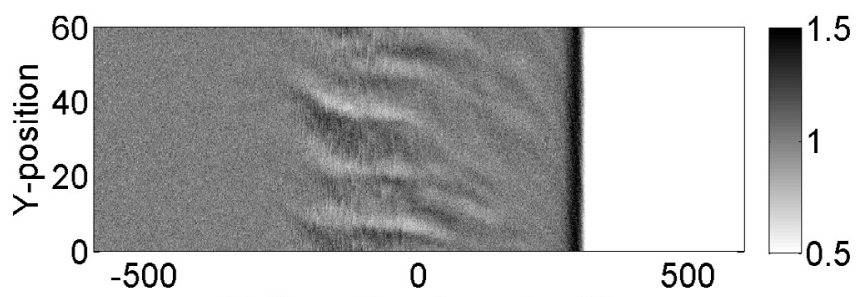

(a) X-position: Ions cloud 1

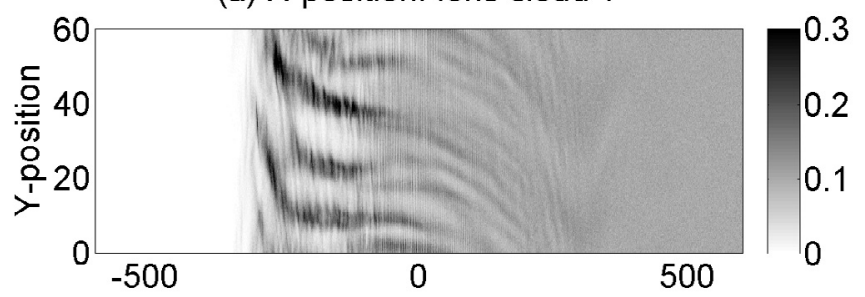

(b) X-position: lons cloud 2

Fig. 4. Ion densities at the time $T_{1}$ normalized to $n_{i 1}$. The ions of cloud 1 are shown in a) and those of cloud 2 in $\mathbf{b}$ ).

qualitative change the fields of the EES undergo as we cross this boundary.

The $B_{y}$ and $B_{z}$ of the EES have a left-hand polarization for $x>300$. Consider a coordinate system defined by $x, B_{y}$ and $B_{z}$. The $B_{y}$ has a maximum at $x \approx 340$, where $B_{z}=0$. As we go to increasing values of $x$, the amplitude of $B_{y}$ decreases and that of $B_{z}$ becomes negative. The magnetic field vector rotates counterclockwise. A deviation from a circular polarization is provided by $B_{z, 0} \neq 0$ and by $E_{x} \neq 0$, but their amplitudes are relatively low compared to $B_{y}$ and $B_{z}$ for $300<x<600$.

The $B_{z}$ is almost in phase with $E_{y}$. The comparison of $B_{y}$ with $E_{y}$ and the comparison of $B_{z}$ with $E_{z}$ for $x>300$ would show that they are shifted by $\approx 90^{\circ}$, while $B_{y}$ is shifted by $180^{\circ}$ relative to $E_{z}$. Figure 2 shows this most clearly at $x \approx 500$. The wave components $\boldsymbol{E}_{\perp}=\left(0, E_{y}, E_{z}\right)$ and $\boldsymbol{B}_{\perp}=\left(0, B_{y}, B_{z}\right)$ perpendicular to $\boldsymbol{v}_{b}$ can thus be connected through $\boldsymbol{E}_{\perp} \approx-\boldsymbol{v}_{b} \times \boldsymbol{B}_{\perp}$, where we assume that $\boldsymbol{v}_{b} \| \boldsymbol{x}$. The EES and its magnetic field is thus convecting with the plasma of cloud 1 . The wavelength of $E_{x}$ in Fig. 2 along $x$ equals that of all field components except $B_{x}$ for $x>300$. Since all components of $\boldsymbol{E}$ are strong, the EES has an electric field component aligned with $\boldsymbol{B}_{0}$. The EES has the wavelength $\lambda_{\mathrm{W}} \approx 120 \lambda_{\mathrm{s}}$, coinciding with $\lambda_{\mathrm{W}} \approx 2 \pi\left(m_{i} / m_{\mathrm{e}}\right)^{1 / 2} \lambda_{\mathrm{s}}$. This $\lambda_{\mathrm{W}}$, together with a convection of the EES with the $v_{b}$ of cloud 1, points at a connection of the EES to phase space structures in the ion distribution, which is typical for SLAMS (Behlke et al. 2003).

The magnetic oscillations observed in Fig. 2 in the spatial interval $-300<x<300$, in which both clouds overlap, must be tied to currents. The currents originate from a spatial redistribution of the plasma cloud particles and the latter can be examined with the help of the density distributions of the four plasma populations.

Figure 4 displays the densities of the ions of cloud 1 and of cloud 2 . The ion density of cloud 1 has a peak at $x \approx 300$. This high-density structure is planar and shows no signs of filamentation along $y$. The filamented front of cloud 2 at $x \approx-300$ shows no high-density structure. The ion densities of both clouds are only strongly modulated well behind the expansion front of cloud 1. Modulations of the density of cloud 1 of up to $40 \%$ are observed for $-200<x<0$ and the density of cloud 2 is modulated even stronger for $-300<x<0$. Weaker modulations of both ion clouds extend up to $x \approx 200$ and their wavelength
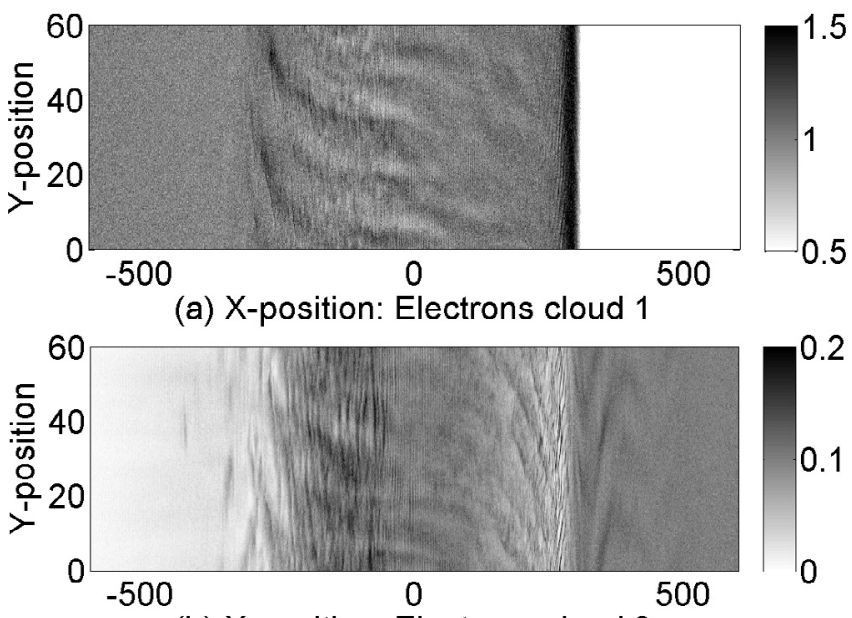

(b) X-position: Electrons cloud 2

Fig. 5. Electron densities at the time $T_{1}$ normalized to $n_{\mathrm{e} 1}$. The electrons of cloud 1 are shown in a) and those of cloud 2 in $\mathbf{b}$ ).

decreases with increasing $x$. The filaments of both ion clouds are aligned with the $x$-axis in the interval $-250<x<-100$. The ion density of cloud 1 is highest at $y \approx 0$ and lowest at $y \approx 10$, while the density of cloud 2 shows the opposite behaviour. The ions of both clouds and their currents are spatially separated, as expected. The ion filamentation takes place on the same scale as the oscillations of $B_{z}$ in Fig. 2c in the same interval, reinforcing their connection. The ion density modulation of cloud 2 oblique to the $x$-axis in the interval $-300<x<-250$ is not balanced by the ions of cloud 1 .

It is remarkable that the amplitude of $B_{z}$ in the interval $-300<x<0$, which displays the strongest ion filamentation, is an order of magnitude lower than that of the EES. The latter must thus have a different origin.

Figure 5 shows the density distributions of the electrons of cloud 1 and 2 . The electrons of cloud 1 accumulate at $x \approx 300$ and their density is modulated on scales of an ion skin depth in the interval $-300<x<0$. Similar structures are revealed by the electron density of cloud 2 in this interval. These patterns follow closely those of the ions in Fig. 4, which is at least partially enforced by the need to satisfy the quasi-neutrality of the plasma. This is evidenced, for example, by the electrons of cloud 1 for $-300<x<-250$. Their density shows the same oblique striping as the ion density of cloud 2 in Fig. 4b. The density maxima of the ions of cloud 2 and of the electrons of cloud 1, as well as their minima, follow each other closely in this interval. It is the electrons, rather than the ions of cloud 1, which compensate the net ion charge in this interval. Another consequence of the quasi-neutrality is seen at the front of cloud 1, where the electrons reach a high density. This density peak is well-defined and confined to a narrow $x$-interval, despite the high thermal electron speed, which should rapidly disperse them. The high ion density in this interval provides the confinement. In contrast, the cloud 2 shows no ion density enhancement in Fig. $4 \mathrm{~b}$ at its front at $x \approx-300$. Consequently, the electrons of cloud 2 are spread out along $x$ and the fastest electrons have reached an $x<-500$.

The ions and electrons of cloud 2 in Fig. $4 \mathrm{~b}$ and Fig. $5 \mathrm{~b}$ show patterns in their density ahead of cloud 1 for $x>300$. These patterns resemble those in $B_{x}$ and $E_{x}$ at $x \approx 300$ in Fig. 2. The box width along $y$ upstream of cloud 1 is approximately one ion skin depth of cloud 2 , because $n_{i 1} / n_{i 2}=10$. The structures just ahead of $x=300$ perform two oscillations along $y$. These are 


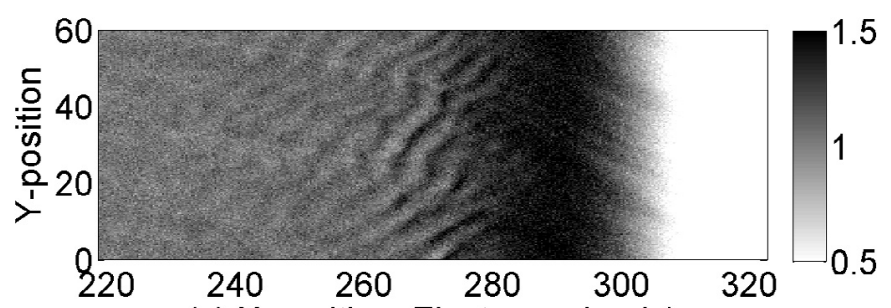

(a) X-position: Electrons cloud 1

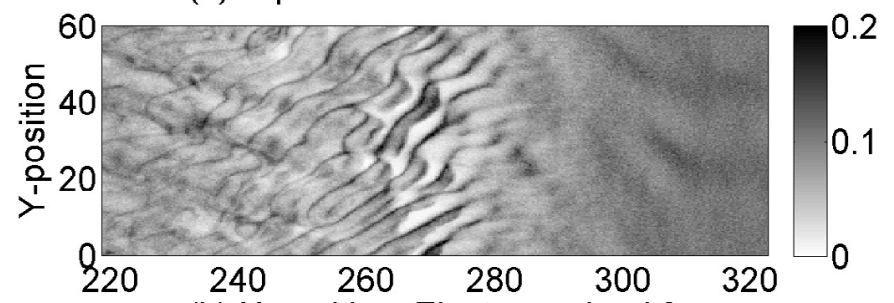

(b) X-position: Electrons cloud 2

Fig. 6. Electron densities at the time $T_{1}$ close to the front of cloud 1 and normalized to $n_{\mathrm{e} 1}$. The electrons of cloud 1 are shown in a) and those of cloud 2 in $\mathbf{b})$.

potentially filaments, but they cannot be caused by the interaction of cloud 1 and 2 because they are outside the cloud overlap layer. The spatial modulation provides a hint. The filament starting at $x \approx 300$ and $y \approx 30$ in Fig. $5 \mathrm{~b}$ changes the value of $y$, at which the density is highest, continuously as a function of $x$. One oscillation takes place on a distance $\approx 120$ along $x$, which is the wavelength $\lambda_{\mathrm{W}}$ of the EES.

The electrons of cloud 2 in Fig. $5 b$ close to the front of the cloud 1 at $x \approx 300$ are also modulated on a smaller scale, which is already evident from Fig. 5. Figure 6 concentrates on this interval. Filaments can be seen with a thickness ranging from $\lambda_{\mathrm{s}}$ to about $5 \lambda_{\mathrm{s}}$ close to $x \approx 280$ and they have vanished already a few tens of $\lambda_{\mathrm{s}}$ behind the front of cloud 1 . Their size and even more so their short lifetime due to a rapid thermalization indicates that these structures are caused by a filamentation of the electrons. Figure $4 \mathrm{~b}$ confirms this, because the large ion filaments form far behind the front of cloud 1, implying that the ions have not been thermalized by their passage through the interval depicted by Fig. 6 .

The densities of the four species in Fig. 7 are integrated over $y$. The plasma density of cloud 1 reaches 1.5 times its initial value at its front at $x \approx 300$ and it rapidly vanishes for further increasing $x$. No ions of cloud 1 and almost none of its electrons have thus propagated upstream of its front. The plasma density of cloud 2 is almost constant for $x>300$. The electron gyroradius in $\boldsymbol{B}_{\perp}$ is less than $\lambda_{\mathrm{s}}$ and they should fall behind the ions of cloud 2 once they encounter the EES. One may expect that the electrons of cloud 2 accumulate at this position, this is however not seen in Fig. 7. The absence of electrons of cloud 1 at this position and the need to satisfy the plasma quasi-neutrality transports the electrons of cloud 2 across $\boldsymbol{B}_{\perp}$, which will accelerate these electrons orthogonally to $x$.

The ion density at the front of cloud 2 and the electron density of cloud 1 at $x \approx-300$ are weakly enhanced. These increased densities are caused by the oblique structures in the interval $-300<x<-250$ in Figs. $4 \mathrm{~b}$ and $5 \mathrm{a}$. The velocity vectors of the ions moving in the oblique filaments have been deflected away from $\boldsymbol{v}_{b}$. This decreases their flow speed along $x$ and the ions accumulate.

The electron densities of both clouds are nonuniform within $-600<x<300$, evidencing their interactions through instabilities. The density dip of the electrons of cloud 2 for

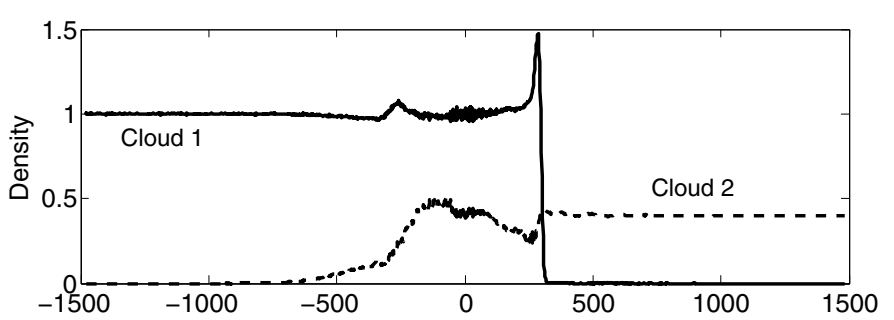

(a) X-position: Electrons

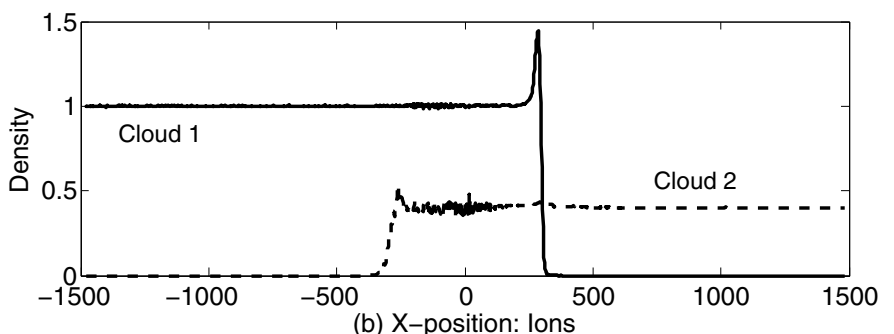

Fig. 7. Densities at the time $T_{1}$ integrated over $y$. a) shows the electron density and b) that of the ions. The dashed curves correspond to the electrons and ions of the cloud 2 and their densities are multiplied by the factor 4 .

$250<x<300$ coincides with the filamentary structures in the same interval in Fig. 6, further evidencing that this dip is caused by an electron filamentation instability without a significant involvement of the ions. A notable fraction of the electrons of cloud 2 have convected to $x \approx-600$ and some even beyond that. The densities of both ion clouds do not yet show a significant modulation, apart from the accumulation at $x \approx 300$. The ions in the layer within $-300<x<0$ in Fig. 4 are only redistributed in the $x, y$-plane, but they do not accumulate along $x$ through a shock compression.

The interaction of the particles with the electromagnetic fields implies that their dynamics involves at least one spatial and three momentum dimensions. We display the phase space projections, which we consider to be the most relevant ones. The ion phase space density $f_{i}\left(x, p_{x}\right)$ can be used to determine if and when the shock forms. The ions of cloud 1 and 2 must merge to form the downstream region. The electron distribution $f_{\mathrm{e}}(x, \gamma)$ shows the range of Lorentz factors attained by the electrons.

Figure 8 displays these distributions. The ions of cloud 1 convect practically freely. Only the tip of this cloud at $x \approx 300$ is modified. The electron gyrofrequency $\omega_{\mathrm{ce}}=e\left|\boldsymbol{B}_{0}\right| / m_{\mathrm{e}}$ equals $\Omega_{\mathrm{e} 1}$, with which we have normalized the time. The ions have gyrated $T_{1} / 400 \approx \pi$ or one half of a gyro-orbit in $\boldsymbol{B}_{0}$ until now. The uniform $\boldsymbol{B}_{0}$ is, however, almost parallel to $x$ and it cannot cause by itself the ion rotation at the front of cloud 1 . The magnetic field $B_{\perp}=\left(B_{y}^{2}+B_{z}^{2}\right)^{1 / 2}$ of the EES exceeds $B_{x, 0}$ at the front of cloud 1 . The ions will have gyrated by a significant angle in this $\boldsymbol{B}_{\perp}$, because the amplitude of the EES grows rapidly to a large amplitude. The EES also accelerates the electrons of cloud 2 in the interval $300<x<500$, which are transported across $\boldsymbol{B}_{\perp}$ by the ions of cloud 2 . The ions of both clouds must provide through the EES the energy required to accelerate the electrons. The modification of the ion phase space distribution can thus be attributed to the EES and to the electron acceleration.

Weak oscillations of the mean momentum $\left\langle p_{x}\right\rangle(x)$ can be seen in the ions of cloud 2 in the interval $300<x<600$ in Fig. 8. We can interpret them as follows. The ions of cloud 2 upstream of cloud 1 move through the EES at the speed $\approx-v_{b}$ in the box frame towards decreasing values of $x$. A strong circularly polarized magnetowave with a wavevector aligned 

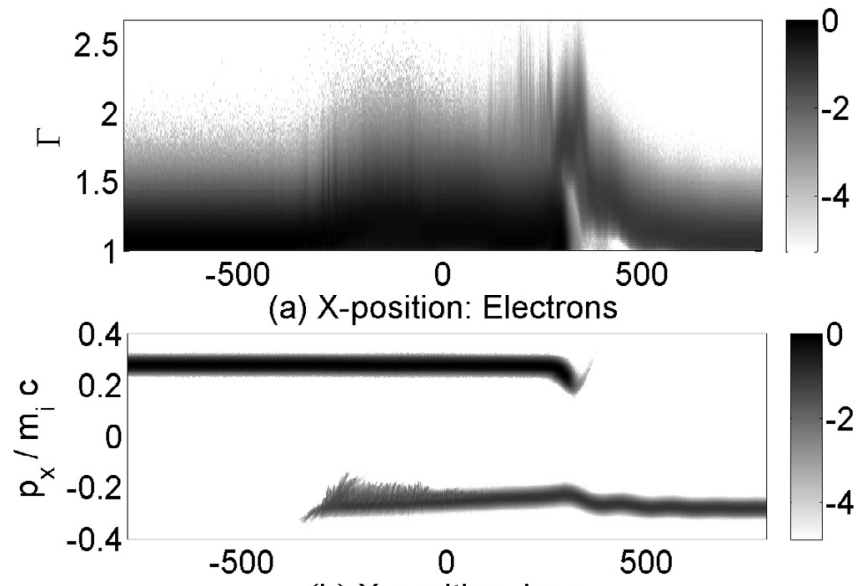

(b) X-position: Ions

Fig. 8. Phase space densities at the time $T_{1}$. a) corresponds to electrons and b) to ions. The greyscale denotes the base 10 logarithm of the density.

with $x$ would force the ions onto a circular path in the $p_{y}, p_{z}$ plane, in which the mean ion beam momenta $\left\langle p_{y}\right\rangle(x)$ and $\left\langle p_{z}\right\rangle(x)$ vary as a function of $x$ with the wave's periodicity. Since $B_{z, 0}$ is not negligible, this motion must also modulate $\left\langle p_{x}\right\rangle(x)$. Finally we see that the ions of the tenuous cloud 2 have been heated up by their filamentation at the front of cloud 2 at $x \approx-300$.

\subsection{Late simulation time}

Unless stated otherwise, we analyse now the simulation data at the time $T_{\text {sim }}=3500$, when we stop the simulation. If both beams had streamed freely, they should have propagated on average the distance $v_{b} T_{\text {sim }} \approx 950$.

Figure 9 displays the densities of both ion clouds. The front of the ions of cloud 1 is located at $x \approx 750$, which is well behind $v_{b} T_{\text {sim }} \approx 950$. The ions of cloud 1 have thus been slowed down and the peak density reaches a value $\approx 6$ at $x \approx 750$. This high-density structure is still planar, but its density is not constant as a function of $y$ any more. It decreases to 4 at $x \approx 750$ and at $y \approx 40$. The front of the ions of the cloud 2 is located at $x \approx-950$, which equals the distance expected from the free streaming of the ions. Both ion clouds reveal density modulations on a scale less or comparable to the ion skin depth. The largest is found in the ions of cloud 2 at $x \approx-300$ and the interval with a reduced density ranges from $y \approx 0$ to $y \approx 30$. The ions of cloud 2 also show structures in the density distribution for $x>750$, which is outside the cloud overlap layer. The wavevector of these oscillations is parallel to $x$.

Movie 1 shows the time evolution of the ion densities until the time $T_{\text {sim. }}$. We first discuss the filamentation in the cloud overlap layer. The filament formation is seen clearly, when each cloud has expanded by the distance 100 or for a time $T_{1} / 3$. The filaments are asymmetric, a consequence of the unequal cloud densities. The ions of cloud 2 show at this time and in the interval $-100<x<0$ several filaments aligned with the $x$-axis. The filaments have a constant density along $y$ and a width $\approx 10$. These filaments are separated by intervals with a reduced density, which have a width of only a few $\lambda_{\mathrm{s}}$. The latter have counterparts in the ions of cloud 1 . The wave vector of these filaments is parallel to the $y$-axis at this time.

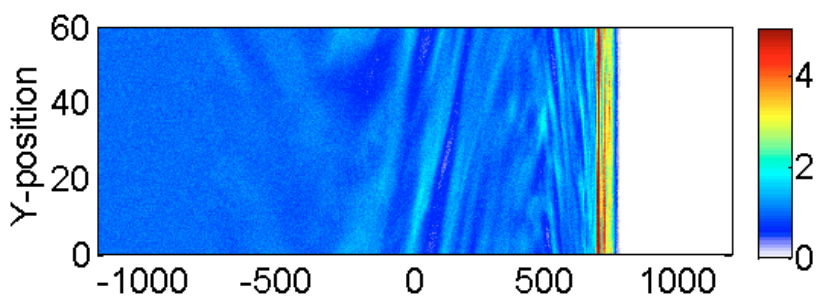

(a) X-position: lons cloud 1

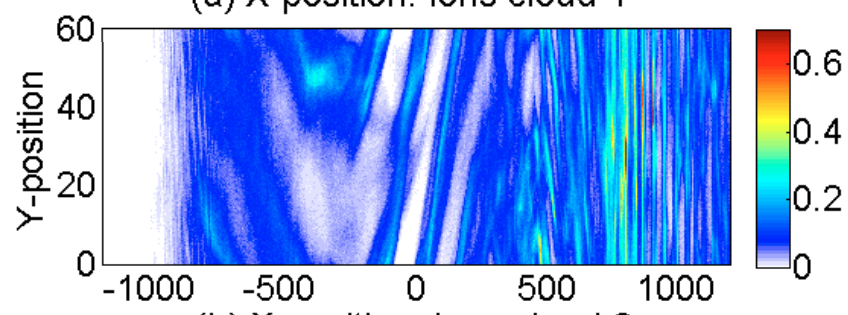

(b) X-position: Ions cloud 2

Fig. 9. (Colour online) Ion density distributions at the time $T_{\text {sim }}$ in units of $n_{i 1}$. The ions of cloud 1 are shown in a) and the ions of cloud 2 in b).

We also observe density fluctuations of the ions of cloud 2 with a wave vector along $x$, which develop first in the section of the cloud overlap layer with $x<0$. This is an instability developing as the ions of cloud 2 propagate through the plasma of cloud 1. A Buneman-type instability (Buneman 1958) develops between the ions of cloud 2, which can be considered to form an unmagnetized beam on electron time scales, and the magnetized electrons of cloud 1 . The phase space density oscillations of the ions of cloud 2 are periodic with a wave length $\lambda \lesssim 4 \pi v_{b} / \Omega_{\mathrm{e} 1}$ (not shown), which is typical for the Buneman instability involving hot electrons. The phase speed of the unstable wave is close to the speed of the ion beam, here composed of the ions of cloud 2, turning it into a slow oscillation in its rest frame. The ions can react to it and they will form phase space holes after a sufficiently long time. The ions of cloud 1 see the Buneman wave as a rapid oscillation to which they cannot react. This explains why the ions of cloud 2 in movie 1 at the time $T_{1}$ and the electrons in Fig. 5 show the density fluctuations, but not the ions of cloud 1 in movie 1 . These charge density fluctuations give the oscillations of $E_{x}$ along $x$ in Fig. 2d. The electrostatic fields probably couple to $B_{x}$, because the Buneman instability is not purely electrostatic in the presence of the strong oblique $\boldsymbol{B}_{0}$.

The ion filaments are observed to move in time in the positive $y$-direction, when the clouds have expanded by the distance 100 along $x$. The flow pattern becomes complicated, when cloud 1 has expanded to $x \approx 400$. The filaments in the interval $0<x<300$ still convect to increasing $y$, while the ion filaments of cloud 2 with $x<0$ move in the opposite direction. We may interpret this observation as follows. Only the ions in the cloud overlap layer are not co-moving with $\boldsymbol{B}_{0}$, because we cannot define through the convection electric field a reference frame, in which the ions of both clouds are at rest. The ions in the cloud overlap layer rotate with a velocity amplitude set by their mean speed relative to the rest frame of the overlap layer, rather than by their smaller thermal speed outside it.

The ions gyrate in the cloud overlap layer orthogonally to $\boldsymbol{B}_{0}$ in a plane that is almost parallel to the $p_{y}, p_{z}$ plane. The ions of cloud 1 and cloud 2 are counter-propagating and the magnetic field should deflect them into opposite directions. The $B_{z}$ separates the filaments of both ion clouds. This implies in a $2 \mathrm{D}$ geometry that the filaments are locked as long as the magnetic trapping 


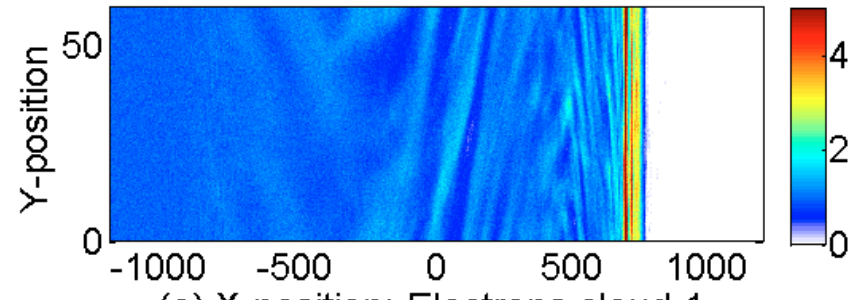

(a) X-position: Electrons cloud 1

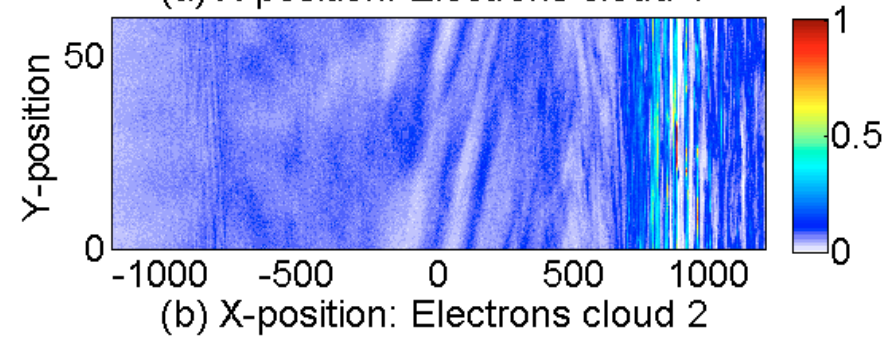

Fig. 10. (Colour online) Electron density distributions at the time $T_{\text {sim }}$ in units of $n_{\mathrm{e} 1}$. a) displays the electrons of cloud 1 and b) the electrons of cloud 2 .

force by $B_{z}$ (Davidson et al. 1972) is stronger than the Lorentz force due to $\boldsymbol{B}_{0}$. A common direction of motion is established. The direction of motion that varies with $x$ and later also with $y$ is probably tied to the ion gyro-phase. Movie 1 suggests at late times that the filaments of cloud 2 close to its front merge and separate again. The $B_{z}$ is here insufficiently strong to separate the ion filaments in the $x, y$ plane. The $\boldsymbol{B}_{0} \neq 0$ introduces a complicated flow pattern in the 5D phase space spanned by $x, y$ and by the three components of $\boldsymbol{p}$. The filaments do probably not merge in this high-dimensional space but they appear to do so in the simulation plane.

Now we turn towards the filaments upstream of cloud 1. As we approach the time $T_{1}$, when the clouds have expanded by the distance 300 along $x$ in the movie 1, the filaments in the cloud overlap region have fully developed and we observe first signs of a filamentation of the cloud 2 ahead of the front of cloud 1 . These filaments are initially oblique as Fig. 4b depicts. When cloud 1 has reached $x \approx 600$, the upstream region ahead of cloud 1 shows strong density oscillations of the ions of cloud 2 along the $x$ axis, which are also depicted in Fig. 9b. They cannot be related directly to the EES, because their wavelength along $x$ is about $\lambda_{\mathrm{W}} / 3$. The patterns and their time-evolution eventually become complicated and presumably also high-dimensional.

The density distributions of the electrons of both clouds are correlated with those of the ions in the overlap region $-950<$ $x<750$, as the Fig. 10 is evidencing. An example is here the density pattern, which crosses $y=0$ at $|x|<100$ and is visible in all four species. The filaments in the interval $-500<x<500$ are, however, more diffuse than those of the ions in Fig. 9. The density peak of the electrons of cloud 1 at $x \approx 750$ is comparable to that of the ions. Again, the electrons are confined to maintain the quasi-neutrality of the plasma. The front end of the electrons of cloud 2 has been replaced by a gradual decrease of the electron density between $-1500<x<-800$. The electrons with $x<-950$ are the fastest ones of cloud 2, which have outrun the bulk of the electrons moving with $-v_{b}$. The ripples in the electron density of cloud 2 at $x>750$ are practically identical to those of the ions in Fig. $9 \mathrm{~b}$.

Movie 2 reveals the time-evolution of the electron densities. Density structures form rapidly close to the initial cloud contact boundary. These structures have no counterpart in the ion distributions in movie 1, until the cloud overlap layer covers $|x| \lesssim 50$. This initial plasma evolution is thus determined by the electron filamentation. The density of the electrons of cloud 1 is enhanced in the cloud overlap layer, while the electron density of cloud 2 is decreased (See also Fig. 7a). Already at this early time, the fastest electrons of cloud 2 outrun the bulk of the electrons to form a low-density tail along $x$ and one consequence is the reduction of the electron density of cloud 2 in the cloud overlap layer. A strong correlation between the ions of both clouds and the electrons of cloud 1 in the overlap layer is observed, when the latter covers $|x| \lesssim 100$, demonstrating that these three species are involved in the formation of the large scale pattern. The electrons of cloud 2 show a distinct behaviour. This is also observed at a later time, when the overlap layer covers $|x| \lesssim 200$. The electrons of cloud 2 are redistributed at this time immediately after they have crossed the front of cloud 1 at $x \approx 200$, which is also shown by Fig. 6, and their density is not visibly correlated with those of the other species.

This behaviour can be understood qualitatively. Consider a dense electron beam and a tenuous electron beam that undergo the filamentation instability, which separates their currents. A highly nonlinear filamentary electron distribution of the tenuous beam can be compensated by a weak perturbation of the dense beam. We thus expect that the spatial density modulations of the tenuous beam become nonlinear first. We also expect, that the high ion inertia results in a delay of their filamentation compared to that of the electrons and both species of cloud 2 will interact differently with the plasma of cloud 1 .

The ions of cloud 2 in Movie 1 show structures similar to those in cloud 1 , which have initially no counterpart in the electrons of cloud 2 (Movie 2). The ions of cloud 2 reveal a higher degree of non-uniformity than the electrons of cloud 1, when the overlap layer spans the interval $|x| \lesssim 200$. We can attribute this to their lower mean density. The increasingly pronounced large-scale modulation of the plasma density of cloud 1 at the time $T_{1}$ imprints itself onto the electrons of cloud 2 . The latter show a modulation on two scales in Fig. 5. The density evolution of the electrons and of the ions eventually becomes similar, except close to the front of cloud 1. Far behind this front, the electrons have thermalized. Since they have no free energy left which could lead to independent behaviour their density distribution follows that of the ions.

We explore now the density evolution of the electrons and ions of cloud 2 ahead of the front of cloud 1 in the movies 1 and 2. We determine now, if we find multiple particle beams upstream of cloud 1 that could give rise to beam-driven instabilities. The electrons of cloud 2 thermalize after they have crossed the front of cloud 1 in Fig. 6 and we can no longer distinguish between the electrons of either cloud. Movie 2 shows no electrons of cloud 1 leaking upstream and the same should hold for the electrons of cloud 2, which have been mixed with those of cloud 1 . This is supported by Fig. 7, which demonstrates firstly that no significant numbers of electrons from cloud 1 diffuse upstream and, secondly, that the electrons of cloud 2 ahead of cloud 1 have a uniform density and convect with the ions to preserve the quasi-neutrality of the upstream plasma. Figure 8 demonstrates that no ions of cloud 1 leak upstream of its front and that no ions of cloud 2 are reflected at $x=300$ to form a beam upstream of cloud 1 . Movies 1 and 2 together with Fig. 2 evidence, however, the growth of the EES and the onset of the filamentation upstream of cloud 1 prior to the time $T_{1}$. These two processes thus grow in an interval, in which we only find the plasma of cloud 2 and no further particle beams. We may thus exclude for our case study and until the time $T_{1}$ that 

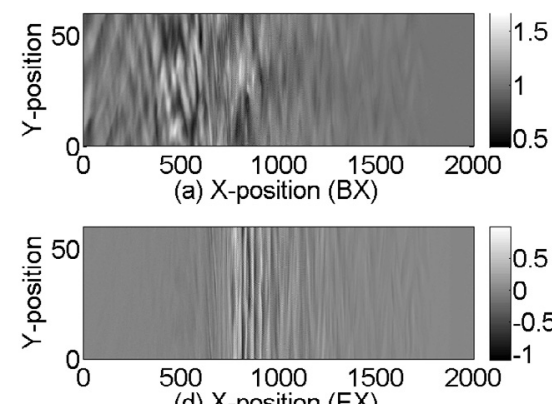

(d) X-position (EX)

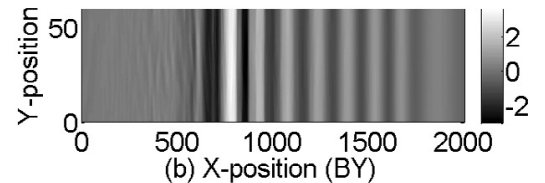

(b) X-position (BY)

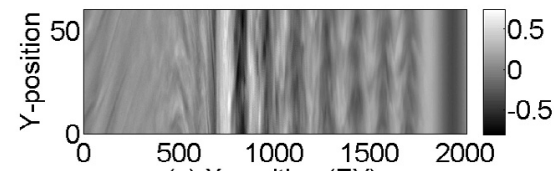

(e) X-position (EY)

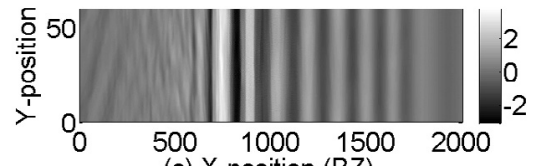

(c) X-position (BZ)

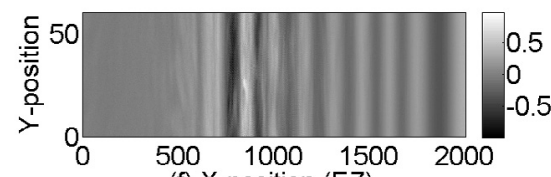

(f) X-position (EZ)

Fig. 11. Electromagnetic field amplitudes at the time $T_{\text {sim. }}$. The upper row shows the magnetic field components $B_{x}$ a), $\left.B_{y} \mathbf{b}\right)$ and $B_{z}$ c). The lower row displays the $\left.E_{x} \mathbf{d}\right), E_{y} \mathbf{e}$ ) and $E_{z} \mathbf{f}$ ).

particle beams (which can be formed by leaking downstream plasma or reflected fast ion beams in a quasi-parallel magnetic field geometry or in unmagnetized plasmas Malkov 1998; Martins et al. 2009), result in the growth of the EES and in the observed upstream instability.

Figure 11 displays the fields at the time $T_{\text {sim }}$. The nonplanar $B_{x}$ reaches amplitudes comparable to $B_{x, 0} / 2$. Both components of $\boldsymbol{B}_{\perp}$ evidence planar oscillations with an amplitude exceeding $B_{x, 0}$ and with a wavelength $\approx 120$. The wavelength is unchanged and the EES is still circularly polarized. The amplitudes of $B_{y}$ and $B_{z}$ peak in the interval $600<x<1000$ close to the front of cloud 1 . Evanescent waves reach far upstream up to $x \approx 1700$. The $\boldsymbol{E}_{\perp}$ is more diffuse and $E_{y}$ shows structuring along $y$. Quasiplanar structures close to and ahead of the front of cloud 1 are revealed by $E_{x}$ for $700<x<1000$. The wave vectors of these oscillations point predominantly along $x$ and their characteristic wavelength is short compared to that of the EES. They resemble the structures of cloud 2 upstream of cloud 1 at late times in the movies 1 and 2 .

The evolution of the fields is presented in the movie 3 for the three components of $\boldsymbol{B}$ and in the movie 4 for the three components of $\boldsymbol{E}$. Until the time $T_{1}$, when the overlap layer covers $|x|<300$, the fields can be subdivided into those in this layer and those of the EES.

We discuss first the electromagnetic fields in the cloud overlap layer. The filamentation is observed best for $B_{x}$ and $E_{y}$. The $B_{z}$ is the dominant component of the filamentation instability in our 2D geometry and for a flow velocity vector along $x$ (Bret et al. 2008; Schlickeiser \& Shukla 2003). This component indeed shows a filamentation, but the adaptation of the colour scale to the EES reduces the contrast and it is not clearly visible. The $B_{x}, E_{y}$ and $B_{z}$ show filamentary structures with a thickness below or equal to the ion skin depth. The filamentation of these field components shows the same flow patterns as the ion distribution in movie 1 . The ion beam filamentation is thus the primary driver of the fields. Oscillations of $E_{x}$ with a short wavelength along $x$ are observed, which we have related previously to a Bunemantype instability.

The EES starts to grow immediately at the cloud collision boundary and it is thus a consequence of our initial conditions. The initial jump of the convection electric field $E_{y}$ across $x=0$, its constant amplitude along $y$ and the vanishing derivative along $z$ imply that $\mathrm{d} E_{y} / \mathrm{d} x=-\partial_{t} B_{z}$. A seed field for magnetowaves develops at the initial contact boundary. We may interpret its consequence as follows. The magnetic field is approximately stationary in the dense plasma. This can be seen from the $E_{y}$ in the cloud overlap layer in Movie 4, which is practically identical to that in cloud 1 outside the layer. The deflection of the electrons of cloud 2 by this $B_{z}$, which is localized in $x$, will be stronger than the deflection of the ions of cloud 2 and $J_{y} \neq 0$. The EES is unstable, which implies that the current amplifies the magnetic perturbation. The EES reaches a $B_{\perp} \approx B_{x, 0}$ already when the cloud overlap layer has expanded to cover $|x| \lesssim 100$. The EES is thus strong enough to cause the deflection of the ions at the tip of cloud 1 at the time $T_{1}$ in Fig. 8. The amplitudes of $B_{y}$ and $B_{z}$ grow and the EES expands steadily until secondary instabilities occur.

When the cloud overlap layer covers $|x| \lesssim 500$, the structures in $B_{x}$ upstream of cloud 1 in movie 3 start to accelerate along $y$. This acceleration is most easily visible close to the position, where the amplitude of the EES peaks. The time, when the structures of $B_{x}$ start to accelerate along $y$, coincides with a similar flow of the structures of $E_{y}$ and $E_{z}$ in movie 4. Prior to their acceleration, the structures in $B_{x}$ resemble the oblique structures in the plasma density of cloud 2 upstream of cloud 1 found at the time $T_{1}$ in Figs. 4 and 5. The density structures and those of $B_{x}$ are modulated along $x$ on scales comparable to the wavelength of the EES.

We may interpret the formation and the acceleration of the field structures in terms of an acceleration of the incoming particles of cloud 2 in the wave field of the EES. If its wave field is not perfectly uniform along $y$, then the particle deflection is not equally efficient for all $y$, which will cause density modulations. The particles of cloud 2 with their spatially nonuniform density are then accelerated in the $y, z$ plane by the EES, resulting in nonuniform currents. A spatially nonuniform current could drive the nonplanar field structures, which we observe in movies 3 and 4. A nonuniform $J_{z}$ would, for example, yield structures in $B_{x}, B_{y}$ and $E_{z}$ by $\partial_{x} B_{y}-\partial_{y} B_{x}=\partial_{t} E_{z}+J_{z}$. The motion of $B_{x}$ and also of $E_{z}$ and $B_{y}$ would then be induced by that of the density structures. The growth and the accelerating motion along $y$ of the structures are presumably tied to the increasing amplitude of the EES.

Movie 4 shows that the $E_{x}$ oscillates outside the cloud overlap layer. The amplitude of $E_{x}$ is constant within each cloud on scales much larger that $\lambda_{\mathrm{s}}$ and the oscillations start instantly. Thus, they cannot be connected to the processes at $x \approx 0$, because of the finite light speed. These oscillations are periodic in time and their frequency is the electron plasma frequency of the respective cloud. These oscillations presumably originate from a slight initial mismatch of the currents of electrons and ions along $x$ in each cloud, which results through $\partial_{t} E_{x}=-J_{x}$ in electrostatic oscillations at the local plasma frequency.

Structures in the plasma of cloud 2 with a wave vector that is aligned with $x$ were observed in Figs. 9 and 10 for $750<$ $x<1200$. Movies 3 and 4 provide additional information. These 


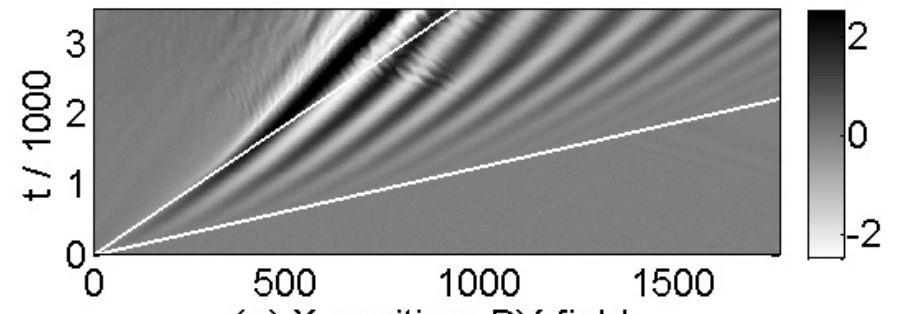

(a) X-position: BY-field

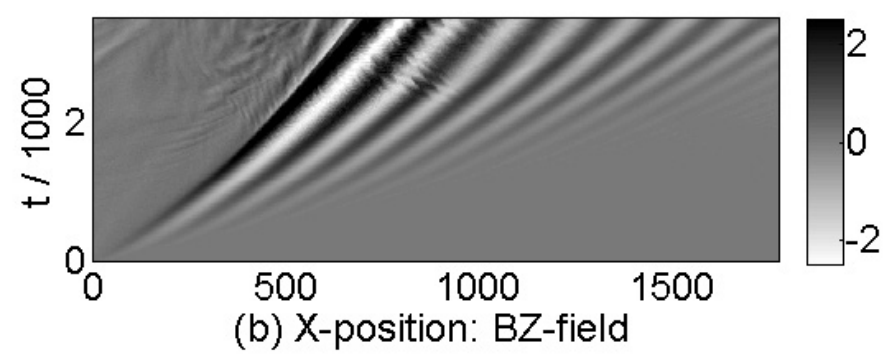

Fig. 12. The $\boldsymbol{B}_{\perp}(x, y=0, t)$ in the reference frame of the simulation box. The $B_{y}$ is shown in a). The two white lines correspond to $x=v_{b} t$ and $x=0.8$ ct. The $B_{z}$ is shown in $\mathbf{b}$ ).

short-scale structures do not show up in $\boldsymbol{B}_{\perp}$ and hence they do not have a strong electromagnetic component. The modulations are most clearly visible from $E_{x}$, for example in Fig. 11d. A polarization along $\boldsymbol{v}_{b}$ indicates that these waves are electrostatic. The density structures in cloud 2 should thus be charge density waves, similar to the Buneman waves we have observed in Fig. 2d. This charge density wave is not the only one upstream of cloud 1. Movie 3 shows at late times and for large $x>0$, that magnetowaves with a longer wavelength than that of the EES are generated that move faster than the EES.

A wave conversion in form of a four-wave coupling of a circularly polarized, large amplitude wave has been proposed by Goldstein (1978). The four interacting waves all have parallel wave vectors and they will thus all result in plasma and field modulations along $x$ in our geometry. The four-wave interaction results in a forward-propagating magnetowave with the same polarization as the driving wave, which might be the long-wavelength magnetowave observed in movie 3 . It is faster than the super-Alfvénic EES, implying that it should propagate in the high-frequency branch of the left-hand polarized mode (Treumann \& Baumjohann 1997). This parametric wave interaction also pumps a charge density wave, which could result in the density modulation observed in the movies 1 and 2 ahead of cloud 1 . Finally, the interaction should produce another magnetowave that moves in the opposite direction than the EES.

We could not identify this fourth wave in $\boldsymbol{E}\left(x, y, t_{0}\right)$ and $\boldsymbol{B}\left(x, y, t_{0}\right)$ at any given time $t_{0}$. This either means it is not there or that its amplitude is close to noise levels. Its wave vector is aligned with that of the EES and it is a magnetowave (Goldstein 1978). It should thus be detectable in slices of $\boldsymbol{B}_{\perp}(x)$ for $y=0$, which we can plot as a function of time. The space-time correlation allows us to identify structures, which are not easily seen from individual time slices. Figure 12 shows them as a function of time. Both components of $\boldsymbol{B}_{\perp}$ show at $x \approx 800$ and $t \approx 2700$ waves propagating antiparallel to the EES. These fast waves originate from processes taking place at the rear end of cloud 2, which are fuelled by the different expansion speeds of electrons and ions into a vacuum. These waves can be seen already before they start to interact with the EES. The $B_{y}$ amplitude is modulated at $x \approx 1700$ and $t \approx 1100$. These waves

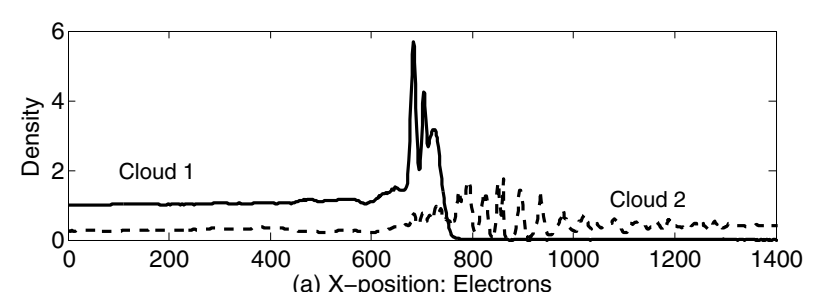

(a) X-position: Electrons

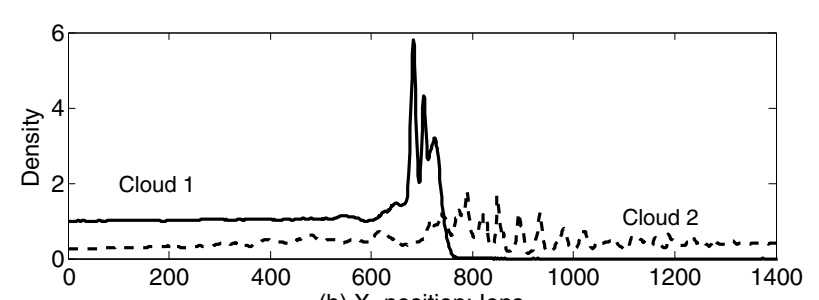

Fig. 13. Densities integrated over $y$ at the time $T_{\text {sim. }}$ a) shows the electron densities and b) the ion densities. The solid curve corresponds to cloud 1 and the dashed curve to cloud 2 . The latter is multiplied by the factor 4 .

are amplified as they pass through the EES. We have to emphasize though, that the backward-propagating magnetowave is only barely visible in this plot and that we have not shown that its amplification by the EES results from a coherent 4-wave interaction. The latter would require more detailed correlation studies of the wave's phases. Our current simulation data is too noisy and we leave this to dedicated 1D PIC studies that can employ a higher number of particles per cell to reduce noise levels.

Two lines are fitted in Fig. 12a. The $x=v_{b} t$ is approximately twice the Alfvén speed $V_{\mathrm{A}}$ of the cloud 2. Initially, the rear end of the EES moves with $v_{b}$ and it is thus connected to the front of cloud 1 . The speed of the EES decreases steadily and its rear end at the time $T_{\text {sim }}$ coincides with the front of cloud 1 at $x \approx 750$ rather than $v_{b} T_{\text {sim }} \approx 950$. This deceleration makes it difficult to determine the frequency of the EES, which should be measured in its rest frame. The first maximum of $B_{y}$ moves with the front of cloud 1 and shows no change in time. We may conclude that its oscillation frequency is low or zero in this decelerating reference frame. The $B_{z}$ shows a change at the front of cloud 1, which may evidence a frequency $\omega_{\text {EES }} \neq 0$ of the EES. It may, however also be related to the formation of the shock. We can thus only conclude here that $\omega_{\text {EES }} \lesssim 2 \pi / T_{\text {sim }} \approx\left(m_{\mathrm{e}} / m_{i}\right) \omega_{\text {ce }}$. The second fitted line in Fig. 12a corresponds to $x=0.8 \mathrm{ct}$ and it is an approximate boundary for the front of the EES. This expansion speed is $0.87 \mathrm{c}$ in the reference of cloud 2 .

Figure 13 shows the densities of both clouds, averaged over $y$, at the time $T_{\text {sim. }}$. We consider only $x>0$. The densities of all four species are practically constant for $x<600$ and the density of each species is comparable to its initial value. The processes in the cloud overlap layer have reached a state that does not involve a density modulation. No shock is present in this interval, because that would result in a plasma compression. The plasma density of cloud 1 rises in the interval $600<x<750$ and decreases to zero for $x>800$. The front of cloud 1 is thus still well-defined. The density of cloud 1 increases to about 3-4 times its initial value in the interval $650<x<750$, reaching a peak density of 6 . The plasma density of cloud 2 reveals substantial oscillations for $x>800$ upstream of cloud 1. The electron and ion density oscillations, which could be observed in the movies 1 and 2, and the $y$-averaged densities do not match perfectly. The 


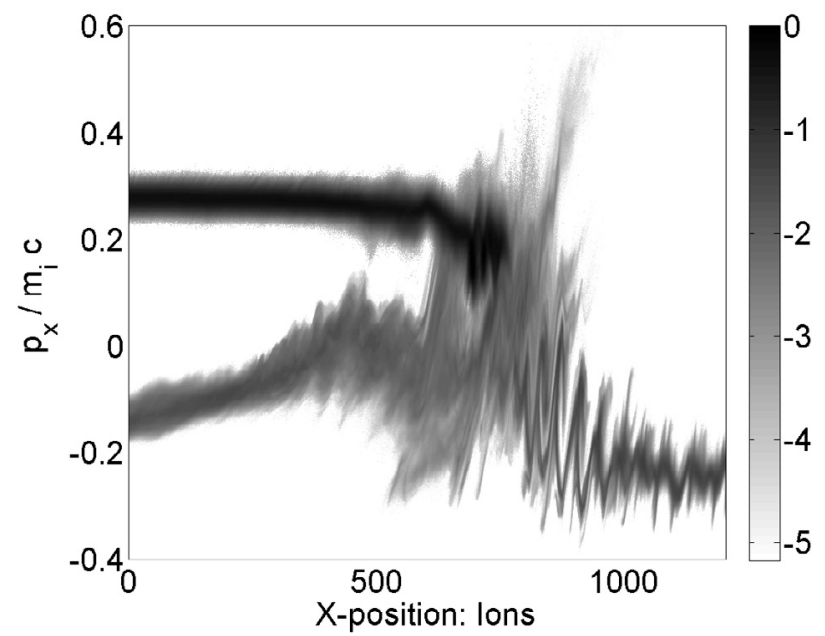

Fig. 14. Ion phase space density at the time $T_{\text {sim }}$. The grayscale shows the 10-logarithmic density normalized to the density peak.

resulting net charge modulation results in charge density waves and the $E_{x} \neq 0$ in Fig. $11 \mathrm{~d}$.

The ion phase space density distribution in the $x, p_{x}$ plane and at the time $T_{\text {sim }}$, which has been integrated over all other position and momentum components, is shown in Fig. 14. The ions of both clouds have started to mix in the $x, p_{x}$-space, which is the condition for a shock formation. The ion clouds merge in the interval $600<x<800$, which coincides with the interval that has an increased plasma density in Fig. 13. The density increase is thus due to the shock compression and it will result in a downstream region that expands along $x$. This future downstream plasma has not yet thermalized, explaining why the density in Fig. 13 is not constant within $600<x<800$.

The forward shock, which will be moving to higher $x$, will be somewhat slower than the collision speed of $c / 2$. It will move faster than $v_{b}$ in the reference frame of cloud 2 . This is because the plasma has a net momentum in the simulation frame due to the higher density of cloud 1 . The shock moving in the direction of decreasing $x$ will move slower than $v_{b}$ in the reference frame of cloud 1. We can already see the slowdown of the front of cloud 1, which coincides with the amplitude maximum of the EES in Fig. 12, which will form the forward moving shock.

Figure 14 also reveals oscillations of the mean momentum $\left\langle p_{x}\right\rangle(x)$ of cloud 2 in the interval $800<x<1000$. The waves that can cause such strong oscillations must yield clearly detectable field oscillations in the same interval. They must be caused either by the charge density waves and the associated $E_{x}$ or by the EES ahead of cloud 1, or by the combination of both. The wavelength of the oscillations is well below the wavelength $\approx 120$ of the EES and comparable to that of the oscillations of $E_{x}$ in Fig. 11d with a wavelength $\lambda_{\mathrm{W}} / 3$. The electrostatic potential of the charge density waves will indeed cause particle oscillations in the $x, p_{x}$ plane. The main effect of the EES, namely the gyrobunching, is not necessarily visible from the phase space density distribution in the $x, p_{x}$ plane. The $B_{\perp}$ of the EES forces the ions on a gyro-orbit orthogonal to $x$. The electrostatic component of the EES provided by $E_{x}$ will, however, modulate the ion distribution in the $x, p_{x}$-plane. No clear modulation of the ion paths on scales $\sim \lambda_{\mathrm{W}}$ is visible in Fig. 14 though.

The electrons are accelerated to highly relativistic speeds in the interval in which we find the charge density waves and the forming shock. The peak Lorentz factor $\gamma$ is 120 , according to

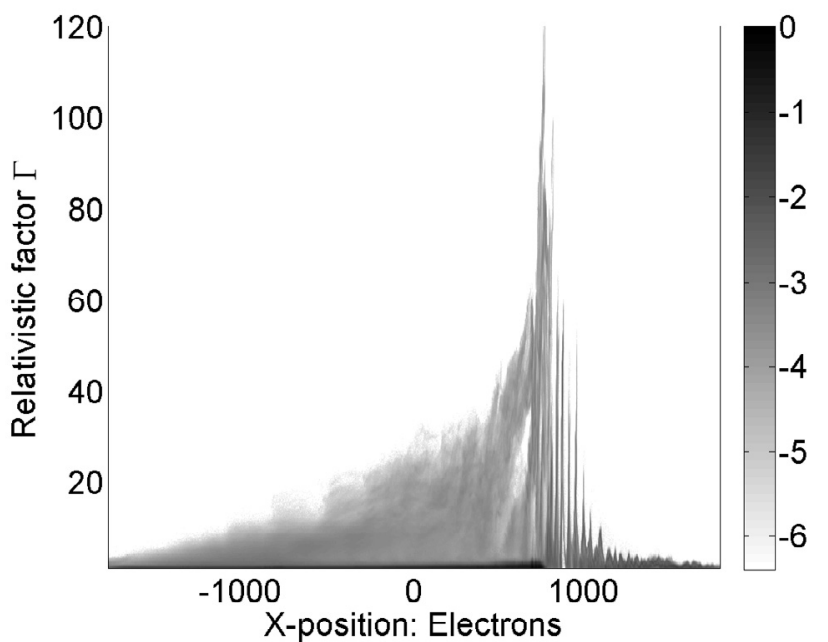

Fig. 15. Electron phase space density at the time $T_{\text {sim }}$. The grayscale is the 10-logarithmic density normalized to its peak.

Fig. 15. This peak Lorentz factor is remarkable in that it exceeds even the $\left(m_{i} / m_{\mathrm{e}}\right)\left(\gamma\left[v_{c}\right]-1\right) \approx 60$, which we would obtain if the energy of an electron would equal that of an ion moving with the cloud collision speed $v_{c}=c / 2$. This energy is, however, reached only by a tenuous subpopulation of the electrons in a limited spatial interval. This strong electron acceleration is presumably achieved by those fields, that give rise to what will become the shock-reflected ion beam. The ions of cloud 2 at $x \approx 750$ in Fig. 14 are accelerated from the speed $-v_{b}$ to the speed $0.5 c>v_{b}$ at this position and their velocity thus changes by more than $v_{c}$. We also observe that the electrons are not only accelerated in the forming downstream region $600<x<800$, but they appear to be pre-accelerated in the interval $800<x<1100$. The electron distribution shows narrow spikes in this region. The electron phase space density is smooth in the interval $500<x<750$ and the energies are here comparable to the energy of an ion moving with $v_{c}$. The peak energy of the electrons decreases steadily, as we go from $x \approx 500$ to $x \approx-1500$. The characteristic distances crossed by electrons with $\gamma>5$ will not differ much during the short time $T_{\mathrm{sim}}$. This distance is thus proportional to the time, which has passed since they were accelerated. The energy to which electrons have been accelerated must therefore have increased with time.

Figure 16 displays the electron phase space density from Fig. 15, which has been integrated over all $x$ to give $N(\gamma)$. It also shows the probability $\tilde{N}(\gamma)=\int_{\gamma}^{\infty} N\left(\gamma^{*}\right) \mathrm{d} \gamma^{*}$ of finding electrons with a Lorentz factor $\gamma^{*}>\gamma$. Most electrons have a $\gamma<5$. The density of this cool population is exaggerated, because we integrate over the full simulation box and, thus, over regions which have not yet been affected by the cloud collision. The high energy tail in the interval $5<\gamma<100$ in both distributions can be approximated by an exponential and the densities decrease rapidly to zero for $\gamma>120$.

Movie 5 provides further insight into the electron acceleration mechanisms. It is a time-animation of the phase space density distributions $f_{i}\left(x, p_{x}\right)$ of the ions and $f_{\mathrm{e}}(x, \gamma)$ of the electrons until the time $T_{\text {sim. }}$. The colour scale denotes the 10-logarithmic number of computational particles in units of those of cloud 2. One particle of cloud 1 corresponds to 10 of cloud 2.

During the initial phase of the cloud collision, up to the time when the ions of both clouds overlap in the interval $|x| \lesssim 50$ in movie 5, the ions propagate practically freely. The ion phase 


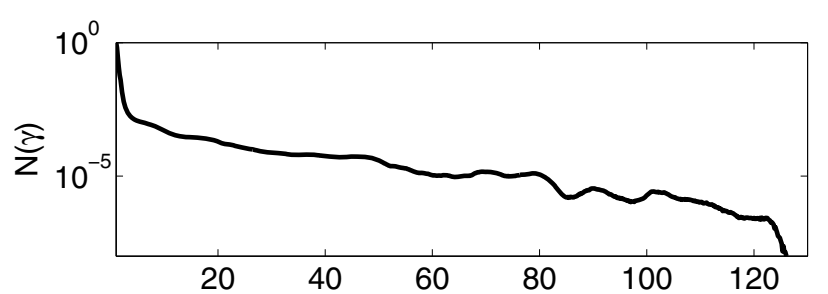

(a) Lorentz factor $\gamma$

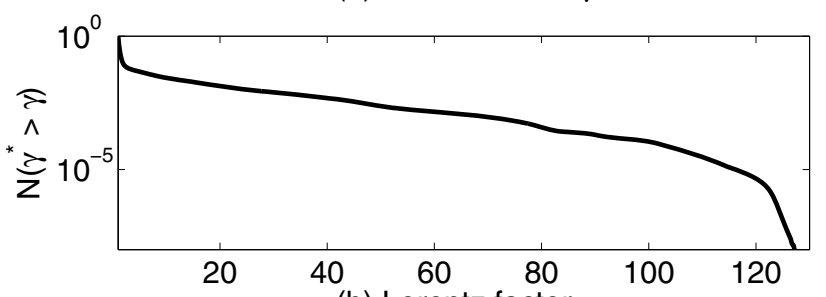

(b) Lorentz factor $\gamma$

Fig. 16. Electron energy distributions, sampled at the time $T_{\text {sim }}$ and normalized to their respective peak value, as a function of $\gamma$. The number density $N(\gamma)$ is displayed in a), while b) shows $\tilde{N}(\gamma) \equiv N\left(\gamma^{*}>\gamma\right)=$ $\int_{\gamma}^{\infty} N\left(\gamma^{*}\right) \mathrm{d} \gamma^{*}$.

space distribution at the front of each cloud is increasingly tilted, because ions with a higher speed propagate farther in a given time interval. The electrons are moderately heated to $\gamma \approx 2.5$ at the front of cloud 2 . The heating mechanisms are the filamentation instability and the Buneman-type instability, which also increase the thermal spread of the ions of cloud 2 at its tip in Fig. 8b.

When the cloud overlap layer covers $|x| \lesssim 300$, the electron heating at the front of cloud 2 has ceased and the electron acceleration sets in at the front of cloud 1 . The slowdown of the ions of cloud 1 is visible and it increases steadily, which is consistent with a magnetic deflection by the growing EES. When the ions of cloud 1 have reached $x \approx 400$, the electrons are accelerated to $\gamma \approx 6$. The movie 5 shows that this electron acceleration is limited to the interval covered by the EES. The electrons of cloud 2 are accelerated as they approach the front of cloud 1. This acceleration results in a beam in $f_{\mathrm{e}}(x, \gamma)$ prior to their encounter with the front of cloud 1 and a depletion of nonrelativistic electrons. The movie 5 shows this as a detaching of the electron distribution from the coordinate axis $\gamma=1$.

We may interpret this as follows. The electrons of cloud 2 are dragged with the ions across the $\boldsymbol{B}_{\perp}$ of the EES and all electrons experience the same accelerating force during the same time interval, resulting in a similar velocity increment for all electrons. The initial electron beam stays compact and the electrons move on a corkscrew orbit in the circularly polarized EES (Dieckmann et al. 2008b). After this acceleration, the electrons of cloud 2 cross the front of cloud 1 and they are mixed in phase space to form a smooth density distribution. Movie 5 demonstrates that the electron acceleration by the EES increases steadily in time, presumably because their cross-field transport across the EES with its increasing amplitude and extent along $x$ provides a stronger acceleration for a longer time.

When the ions of cloud 1 have propagated to $x \approx 500$ in movie 5, the ions of both clouds start to merge. We show the phase space densities $f_{i}\left(x, p_{x}\right)$ and $f_{\mathrm{e}}(x, \gamma)$ of both species directly after this merger at the time $0.8 T_{\text {sim }} \approx 2800$ in Fig. 17 . We notice at this time localized and strong oscillations of $\left\langle p_{x}\right\rangle(x)$ at $800<x<1000$ and weaker ones with a shorter wavelength within the interval $400<x<800$. The electrons are accelerated to highly relativistic energies by these structures, as expected.
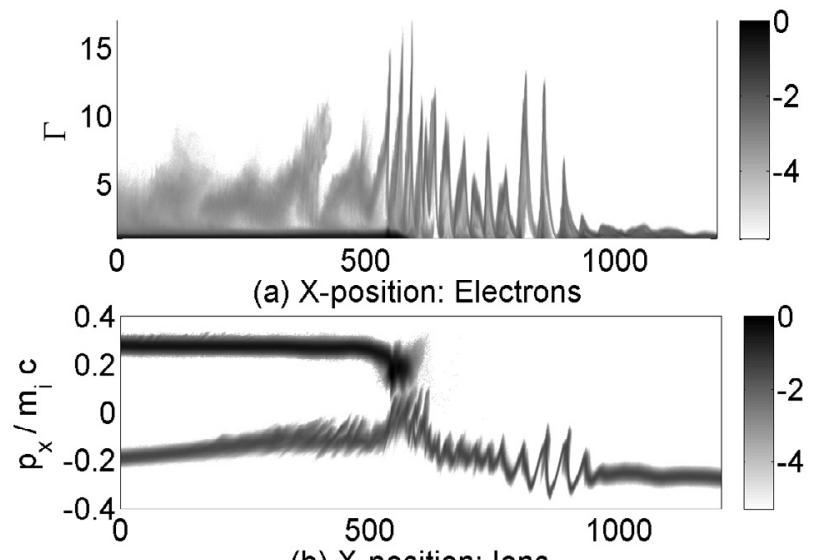

(b) X-position: Ions

Fig. 17. Phase space distributions at the time $0.8 T_{\text {sim }}$. a) displays the electrons and $\mathbf{b}$ ) the ions.

Consider a force that causes velocity oscillations of the ions of $\Delta V \approx 0.1 c$ in Fig. 17b. The same force will clearly result in relativistic electron velocity oscillations. The oscillations of the ion mean momentum move in the negative $x$-direction in movie 5 and they are amplified as they pass through the EES.

We may associate these wave structures with the backward propagating charge density waves and magnetowaves expected from the four-wave interaction, although we have to point out that the strong plasma modulations imply a nonlinear stage of the plasma, which is not incorporated into the equations describing the four-wave interaction. A consequence can be that e.g. the strong and compact magnetowave modulates the plasma density by the ponderomotive force of its fields. The fields resulting in the large modulation must belong to a well-defined wave packet. Figure 12 evidences at the time $0.8 T_{\text {sim }}$ the presence of a magnetowave in the interval $800<x<1000$, in which we find the strong momentum oscillation of cloud 2 in Fig. 17b. The oscillations of $\left\langle p_{x}\right\rangle(x)$ in the interval $600<x<700$ may correspond to the charge density wave, which we observed at late times in the movies 1 and 2. Both, the charge density waves and the magnetowave accelerate electrons to highly relativistic speeds.

We finally assess the energy contained by the fields at the time $T_{\text {sim }}$ and the value of plasma collisions of the type we have considered here for the magnetic field amplification upstream of the main blast shell of a SNR. The magnetic energy density in physical units $\boldsymbol{B}^{2} / 2 \mu_{0}$ is normalized to $\boldsymbol{B}_{0}^{2} / 2 \mu_{0}$ and the electric energy density in physical units $\epsilon_{0} \boldsymbol{E}^{2} / 2$ is normalized to $c^{2} \boldsymbol{B}_{0}^{2} / 2 \mu_{0}$. Both are plotted in Fig. 18. Significant field energy densities are found only close to the front of cloud 1 at $x \approx 750$. The magnetic field dominates and its peak energy density exceeds that of the electric field by the factor $\approx 25$. The slowdown of the front of cloud 1 implies that the $\boldsymbol{E}_{\perp}=-\boldsymbol{v}_{f} \times \boldsymbol{B}_{\perp}$ decreases, where $\boldsymbol{v}_{f}$ is the speed of the front of cloud 1 that was initially $\boldsymbol{v}_{b}$. The energy density of the magnetic field is amplified by over an order of magnitude by the plasma collision and by the shock formation. The strongest magnetic fields corresponding to the circularly polarized EES coincide with the fastest electrons in the simulation. This interval should emit significant electromagnetic radiation.

\section{Discussion}

We have described in this paper the collision of two plasma clouds at the speed $\mathrm{c} / 2$. The ion to electron mass ratio of 400 has 

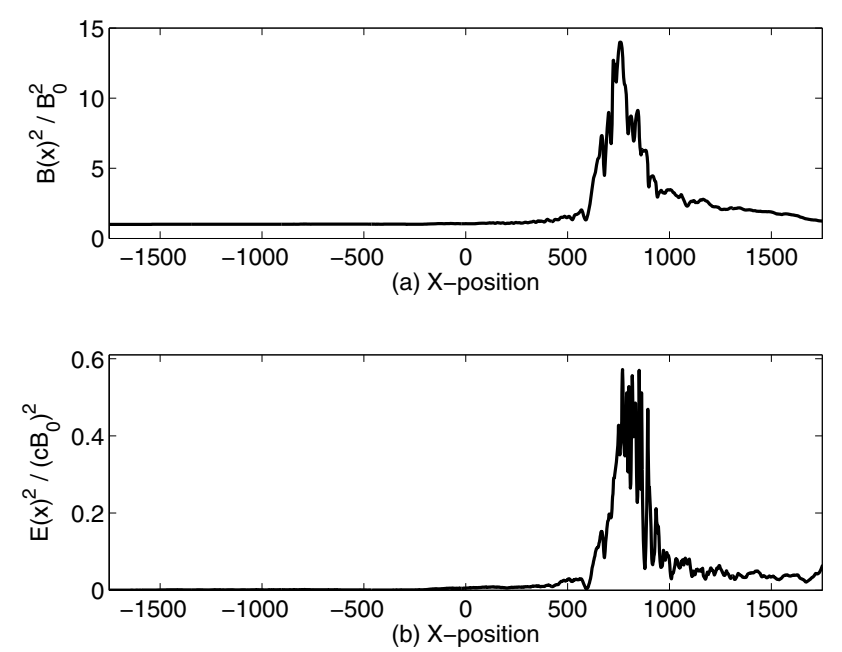

Fig. 18. Field energy densities at the time $T_{\text {sim }}$. a) considers the magnetic energy density and b) that of the electric field.

allowed us to model the collision in two spatial dimensions until the shock forms. Then we had to stop the simulation. The acceleration of electrons to speeds $\sim c$ and the rapid expansion speed of the energetic electromagnetic structure (EES) imply, that both will quickly reach the boundaries; hence our periodic boundary conditions become invalid. Open boundaries would allow the electrons and the wave energy to flow out of the system. However, the instabilities driven by these beams (Martins et al. 2009) and by the EES are important for the upstream dynamics and the latter will be adversely affected by open boundaries.

The filamentary structures can form and merge in the 2D geometry we consider here up to the instant when the magnetic repulsion of two filaments with oppositely directed current enforces their spatial separation (Davidson et al. 1972; Lee \& Lampe 1973), at least in the absence of an oblique magnetic field. Then no further mergers occur since only one dimension is available orthogonal to the flow velocity vector. A realistic 3D PIC simulation would allow the filaments to move around each other and merge with other filaments of equal polarity (Lee \& Lampe 1973). A 3D PIC simulation is, however, currently impossible for our case study involving ions, because of the computational cost involved in resolving ion and electron scales. Simulations in three spatial dimensions are now feasible for the case of leptonic shocks (Nishikawa et al. 2009).

The densities of the clouds differ by a factor of 10 and the collision is asymmetric, as in the simulation by Bessho \& Ohsawa (1999); Zindo et al. (2005); Sorasio et al. (2006). Initially the magnetic field is uniform and quasi-parallel to the flow velocity vector. Its significant strength together with the high plasma temperature of $25 \mathrm{keV}$ and the unequal cloud densities reduce the growth rate of the filamentary instabilities (Bret et al. 2006, 2007).

Dieckmann et al. (2008b) have previously probed the higher $\gamma$ regime appropriate for the internal shocks of gamma-ray bursts. Here we consider the mildly relativistic regime, with a collision speed $0.5 c$ between both clouds. Such a speed might be realistic for a plasma subshell outrunning the main SNR shock. Such subshells can reach relativistic flow speeds for particularly violent SNR explosions (Kulkarni et al. 1998). In our initial conditions, the magnetic energy surpasses the thermal energy of the dense plasma slab by a factor of 5 . The plasma flow implies, however, that the box-averaged plasma kinetic energy density exceeds the magnetic energy density by an order of magnitude. We summarize several aspects of our results.

\subsection{Effects due to initial conditions}

Our initial conditions have resulted in the formation of planar wave and plasma structures, the most important one being the EES. We think that the EES grew out of a localized seed magnetic field pulse driven by the spatial gradient of the convection electric field. The plasma upstream of the dense cloud is destabilized by this electromagnetic structure and the EES expands at the speed $0.87 c$ in the reference frame of the tenuous cloud. The energy for its growth and expansion is provided by the kinetic energy of the upstream medium, which moves with respect to the EES. The shock speed $\lesssim v_{c}$ then implies that we have a coherent magnetic layer that expands its width at a speed of at least $\left(0.87 c-v_{c}\right) /\left(1-0.87 v_{c} / c\right) \approx 0.65 c$, measured in the reference frame of the tenuous cloud. It covered about 80 ion skin depths at the end of the simulation, showing no signs of a slowdown.

The EES is a consequence of our initial conditions and the growth of the seed magnetic field amplitude could probably be reduced but not suppressed by a smoother change of the convection electric field, which can be achieved by a gradual change of the plasma convection speed (Bessho \& Ohsawa 1999; Zindo et al. 2005). However, the seed magnetic field could be provided also by waves with a short wavelength, e.g. whistlers, and it is thus not unphysical.

Structures with strong magnetic fields, similar to the EES and known as SLAMS, are frequently observed close to quasiparallel shocks in the solar system plasma and they can accelerate electrons to high energies. They are thus potentially important also for SNR shock physics. Our initial conditions provide a possibility to let nonlinear MHD waves grow out of a simple simulation setup for a further study. The EES is moving with the ions of the dense cloud and it modulates the ions and electrons of the tenuous cloud, thereby gaining energy. Its growth probably requires an asymmetric plasma collision.

\subsection{Shock formation}

In this paper we modelled the formation of the shock from the initial collision of two plasma clouds. The signatures of the shock are evident, including visible thermal broadening behind the shock and a dense shock ramp. While filamentation structures form ahead of and behind the shock, we note that the structure is basically planar in the critical foreshock area, where electron acceleration is expected to occur. This means that onedimensional simulations will be relevant in this region, allowing much higher resolution, increased particle number (resulting in lower particle noise and a better phase space resolution) and a higher ion-electron mass ratio, than is currently found in two and three-dimensional simulations.

It is evident from the simulation that the filamentation is not fully suppressed by the guiding magnetic field and by the high plasma temperature. Its amplitude has been set such that it should suppress the electron filamentation if the plasma would be spatially uniform (Bret et al. 2006). This amplitude is apparently insufficient to suppress the slower filamentation of the ion beams and we could even see evidence for an electron beam filamentation just behind the front. The front of the dense plasma cloud maintains its planarity throughout the simulation, but even here the density was non-uniform along the boundary. The onset of the filamentation was, however, delayed. The likely cause is 
the high density gradient across the front, which alters the electron and ion skin depths and thus the characteristic scale of the filaments. The gradient is caused by the slowdown of the ions by the magnetic field of the EES and by the electron acceleration. The electrons are confined at the front in the direction of the shock normal so that they preserve the quasi-neutrality of the plasma, but they can move orthogonally to it. The latter results in a drift current.

\subsection{Field amplification}

Volk et al. (2005) and Ellison \& Vladimirov (2008) have described observations of magnetic field amplification above the value expected from shock compression in SNRs. At the final simulation time the magnetic field energy density is increased in strength by over one order of magnitude, exceeding by far that expected from the magnetic field compression by the shock. A shock compresses only the magnetic field component perpendicular to the shock normal, which is weak in our case, and the amplification of its energy density can reach a factor of 4-7. The magnetic energy density at the simulation's end has been comparable to the box-averaged kinetic energy density in an interval spanning about 10-20 ion skin depths. The magnetic energy density due to the EES was at least twice as high as that of the background field in an interval covering 50 ion skin depths. Even if we take into account that the kinetic energy density close to the shock is increased by the accumulation of plasma, the magnetic energy density still constitutes a sizeable fraction of the local total plasma kinetic energy density. The EES has provided the main contribution to the magnetic energy density and exceeded that due to the filaments downstream by two orders of magnitude.

Throughout this paper, we used normalized quantities in our simulation and we can scale the magnetic field amplitude to the relevant plasma conditions. If we set the electron density of the dense cloud to $1 \mathrm{~cm}^{-3}$, we would obtain a peak magnetic field with a strength of $10 \mathrm{mG}$. However, we have to point out that our initial magnetic field amplitude has been higher than that expected for the ambient plasma, even if we take into account its amplification by cosmic ray-driven instabilities, and our simulation results may not be directly applicable.

Where does the extra field come from? Amplification of the magnetic field can occur from the electron drift current arising from the $\boldsymbol{E} \times \boldsymbol{B}$ drift motion in a layer close to the shock that is narrower than the ion gyroradius but wider than the electron gyroradius, see, e.g. Baumjohann \& Treumann (1996). The current adds to the shock current and increases the jump in the perpendicular magnetic field. This can only occur when the ion and electron gyroradii differ, i.e. not in a pair plasma. The EES has a significant $E_{x}$-component and $\left|\boldsymbol{B}_{\perp}\right| \approx\left|\boldsymbol{B}_{0}\right|$. We thus obtain a $\boldsymbol{E} \times \boldsymbol{B}$ drift orthogonal to the flow velocity vector.

We have also found that the requirement to maintain quasineutrality of the plasma implies that the upstream electrons are dragged with the upstream ions across the EES, which moves with the shock. The resulting $\boldsymbol{v} \times \boldsymbol{B}$ drift accelerates the electrons orthogonally to the shock propagation direction, further enhancing the net current and the magnetic field. Finally, magnetic fields of SLAMS are provided by the current due to the gyro-bunched ions, which rotate in the plane orthogonal to the wavevector.

These mechanisms increase the mean magnetic field, and are different from the instability described by Bell (2004) who has described a cosmic ray streaming instability which can amplify turbulent magnetic fields ahead of the shock. We can exclude
Bell's instability here since we have not found energetic particles with a significant density moving upstream, which would provide the net current that drives this instability.

\subsection{Electron acceleration and upstream wave spectrum}

The shock retains its planar structure after it forms. A circularly polarised large-scale precursor wave, the EES, expands into the foreshock. Its wavelength is several times the ion skin depth. It gradually rotates the quasi-parallel magnetic field into a quasiperpendicular one at the current layer and it forces the incoming ions and electrons to interact with it nonlinearly. The ions are gyro-bunched and some of the incoming ions of the tenuous cloud are reflected by the forming shock. We have found evidence of a parametric instability (Goldstein 1978) of the EES ahead of the foreshock and we could find at least two waves that may be the result of this parametric decay. These waves appear at late times, when the EES has expanded in space and is thus sufficiently monochromatic. They grow to an amplitude that introduces oscillations of the mean velocity of the ions of up to $c / 5$.

The interplay of the short-scale charge density waves and magnetowaves causes the electrons to be accelerated to highly relativistic speeds upstream of the forming shock. Similar electron acceleration (injection) mechanisms upstream of shocks involving whistler waves have been proposed by Levinson (1992); Kuramitsu \& Krasnoselskikh (2005a,b). The strongest electron acceleration is, however, observed at the location where the shock-reflected ion beam is developing. The electrons are accelerated to a peak Lorentz factor of 120 and their energy gain is thus comparable to the energy associated with the velocity change of the shock-reflected ions. If the electron acceleration is accomplished by the electromagnetic fields that reflect the incoming upstream ions, then the energy gain of the electrons may scale with the ion mass. We may expect in this case that the electrons are accelerated to a Lorentz factor $\gamma_{\mathrm{M}} \approx 120 m_{p} / m_{i}$ that is $\gamma_{\mathrm{M}} \approx 550$ if we would use the correct proton to electron mass ratio.

It is interesting to see if this type of electron acceleration can also occur close to Solar system shocks. Let us consider the Earth bow shock as one of the best known collisionless shocks and let us assume a Solar wind speed of $4 \times 10^{5} \mathrm{~m} / \mathrm{s}$ to $7.5 \times 10^{5} \mathrm{~m} / \mathrm{s}$. A specular reflection of the incoming Solar wind protons by the bow shock would change their energy by about $0.8 \mathrm{keV}$ to $3 \mathrm{keV}$. Electrons with such energies are observed in a thin sheet close to the shock surface of perpendicular shocks (Anderson et al. 1979). Perpendicular shocks are capable to produce shock-reflected ion beams (Lembege et al. 2004) and the electron acceleration mechanism we observe here may work also at the Earth bow shock.

\subsection{Future work}

This simulation study was concerned with the collision of two plasma clouds at a mildly relativistic speed. Its purpose has been to better understand the conditions and the mechanisms involved in the formation of a shock. This shock will move at an essentially nonrelativistic speed below the initial collision speed and, thus, be relevant for fast SNR flows. A two-dimensional simulation geometry was necessary to assess the importance of the multi-dimensional filamentation instability for the shock dynamics. A quasi-parallel guiding magnetic field was used to slow down this filamentation, resulting in a planar (one-dimensional) shock. 
The formation of the shock could be observed, but the simulation had to be stopped at this time due to computational constraints. This simulation has, however, revealed several aspects that should be examined in more detail in more specialised simulation studies.

The EES probably formed in response to our initial conditions. i.e. the sharp jump in the convection electric field at the cloud collision boundary. It has to be investigated if the EES also forms if this jump is decreased, for example by a higherorder field interpolation scheme or by a gradual decrease of the convection electric field by a smooth change in the plasma convection speed. The simulations by Bessho \& Ohsawa (1999) and Zindo et al. (2005) suggest that this will leave unchanged the magnetic field amplification and the electron acceleration. However, the EES may not be so strong and coherent.

The magnetic field amplitude in the present study is higher than it is realistic for a SNR scenario. Future studies must address how the shock formation depends on lower amplitudes of the guiding magnetic field. Computationally inexpensive parametric simulation studies that resolve only one spatial dimension may provide insight. Initial studies not discussed here indicate that the shock formation is delayed by a decreasing magnetic field amplitude.

It is also necessary to follow the plasma collision for a longer time. An important aspect is here how far the EES can expand upstream and how strong the downstream magnetic field is. The planarity of the EES and of the shock boundary may permit us to use one-dimensional simulations, by which we can expand by at least an order of magnitude the box size along the collision direction.

An one-dimensional simulation also allows us to examine with a larger number of particles per cell and, thus, lower noise levels the secondary instabilities driven by the EES. We have found evidence for an instability of the EES to a four-wave interaction. Lower noise levels would allow us to compare the amplitudes and phases of the EES with those of the secondary waves, which is necessary to demonstrate a coherent wave interaction. Extending the simulation time together with suitable initial conditions would, potentially, allow us to investigate what happens if the speed of the EES decreases below the local Alfvén speed. It is possible that the EES decouples from the shock and propagates independently in form of an Alfvén wave packet.

Finally, it would be interesting to reduce the collision speed to about $c / 10$, which is close to the expansion speed of the SNR shock, to see if and how many electrons are accelerated to relativistic speeds. This will provide insight into the electron injection efficiency of oblique shocks and, thus, into the ability of SNR shocks to accelerate electrons to cosmic ray energies.

Acknowledgements. G.C.M. is funded by the Science Foundation Ireland grant 08/RFP/PHY1694. M.E.D. is funded by Vetenskapsrådet and by the Deutsche Forschungsgemeinschaft through the grant FOR1048. This research was supported in part by the National Science Foundation under Grant No. PHY05-51164. The authors thank Prof. M. Pohl and Dr. J. Niemiec for useful discussions related to the initial conditions. The Swedish HPC2N and the Irish ICHEC have provided the computer time and support.

\section{References}

Acero, F., Ballet, J., \& Decourchelle, A. 2007, A\&A, 475, 883

Amano, T., \& Hoshino, M. 2007, ApJ, 661, 190

Anderson, K. A., Lin, R. P., Martel, F., et al. 1979, Geophys. Res. Lett., 6, 401

Arber, T. D., \& Vann, R. G. L. 2002, J. Comput. Phys., 180, 339

Baumjohann, W., \& Treumann, R. A. 1996, Basic Space Plasma Physics (London: Imperial College Press)
Behlke, R., Andre, M., Buchert, S. C., et al. 2003, Geophys. Res. Lett., 30, 1177 Bell, A. R. 2004, MNRAS, 353, 550

Bessho, N., \& Ohsawa, Y. 1999, Phys. Plasmas, 6, 3076

Bingham, R., Mendonca, J. T., \& Shukla, P. K. 2004, Plasma Phys. Controll. Fusion, 46, R1

Bret, A. 2009, ApJ, 699, 990

Bret, A., Dieckmann, M. E., \& Deutsch, C. 2006, Phys. Plasmas, 13, 082109

Bret, A., Gremillet, L., \& Bellido, J. C. 2007, Phys. Plasmas, 14, 032103

Bret, A., Gremillet, L., Benisti, D., \& Lefebvre, E. 2008, Phys. Rev. Lett., 100, 205008

Buneman, O. 1958, Phys. Rev. Lett., 1, 8

Cargill, P. J., \& Papadopoulos, K. 1988, ApJ, 329, L29

Chapman, S. C., Lee, R. E., \& Dendy, R. O. 2005, Space Sci. Rev., 121, 5

Davidson, R. C., Hammer, D. A., Haber, I., \& Wagner, C. E. 1972, Phys. Fluids, 15,317

Dawson, J. M. 1983, Rev. Mod. Phys., 55, 403

Dieckmann, M. E., \& Bret, A. 2009, ApJ, 694, 154

Dieckmann, M. E., Bret, A., \& Shukla, P. K. 2008a, New J. Phys., 10, 013029

Dieckmann, M. E., Shukla, P. K., \& Drury, L. O. C. 2008b, ApJ, 675, 586

Dieckmann, M. E., Kourakis, I., Borghesi, M., \& Rowlands, G. 2009, Phys. Plasmas, 16, 074052

Drury, L. O. C. 1983, Rep. Prog. Phys., 46, 973

Dupree, T. H. 1963, Phys. Fluids, 6, 1714

Eastwood, J. W. 1991, Comput. Phys. Comm., 64, 252

Ellison, D. C., \& Vladimirov, V. 2008, ApJ, 873, L47

Fransson, C., Chevalier, R. A., Filippenko, A. V., et al. 2002, ApJ, 572, 350

Goldstein, M. L. 1978, ApJ, 219, 700

Hededal, C. B., \& Nishikawa, K. I. 2005, ApJ, 623, L89

Hoshino, M., \& Shimada, N. 2002, ApJ, 572, 880

Katsouleas, T., \& Dawson, J. M. 1983, Phys. Rev. Lett., 51, 392

Kirk, J. G., \& Dendy, R. O. 2001, J. Phys. G., 27, 1589

Kulkarni, S. R., Frail, D. A., Wieringa, M. H., et al. 1998, Nature, 395, 663

Kuramitsu, Y., \& Krasnoselskikh, V. 2005a, Phys. Rev. Lett., 94, 031102

Kuramitsu, Y., \& Krasnoselskikh, V. 2005b, A\&A, 438, 391

Lee, R., \& Lampe, M. 1973, Phys. Rev. Lett., 31, 1390

Lembege, B., Giacalone, J., Scholer, M., et al. 2004, Space Sci. Rev., 110, 161

Levinson, A. 1992, ApJ, 401, 73

Malkov, M. A. 1998, Phys. Rev. E, 58, 4911

Mann, G., \& Classen, H. T. 1997, A\&A, 322, 696

Mann, G., Luhr, H., \& Baumjohann, W. 1994, J. Geophys. Res., 99, 13315

Mann, G., Classen, H. T., Motschmann, U., Kunow, H., \& Droge, W. 1998, A\&SS, 264, 489

Marcowith, A., Lemoine, M., \& Pelletier, G. 2006, A\&A, 453, 193

Martins, S. F., Fonseca, R. A., Silva, L. O., \& Mori, W. B. 2009, ApJ, 695, L189

McClements, K. G., \& Fletcher, L. 2009, ApJ, 693, 1494

Miteva, R., \& Mann, G. 2007, A\&A, 474, 617

Niemiec, J., Pohl, M., Stroman, T., \& Nishikawa, K. 2008, ApJ, 684, 1174

Nishikawa, K. I., Niemiec, J., Hardee, P. E., et al. 2009, ApJ, 698, L10

Ohira, Y., \& Takahara, F. 2007, ApJ, 661, L171

Pelletier, G., Lemoine, M., \& Marcowith, A. 2006, A\&A, 453, 181

Raadu, M. A., \& Rasmussen, J. J. 1988, Ap\&SS, 144, 43

Reville, B., Kirk, J. G., Duffy, P., \& O'Sullivan, S. 2007, A\&A, 475, 435

Rieger, F. M., \& Duffy, P. 2006, ApJ, 652, 1044

Riquelme, M. A., \& Spitkovsky, A. 2009, ApJ, 694, 626

Schlickeiser, R., \& Lerche, I. 2007, A\&A, 476, 1

Schlickeiser, R., \& Lerche, I. 2008, A\&A, 485, 315

Schlickeiser, R., \& Shukla, P. K. 2003, ApJ, 599, L57

Scholer, M., \& Matsukiyo, S. 2004, Ann. Geophys., 22, 2345

Scholer, M., Kucharek, H., \& Shinohara, I. 2003, J. Geophys. Res., 108, 1273

Sircombe, N. J., Dieckmann, M. E., Shukla, P. K., \& Arber, T. D. 2006, A\&A, 452,371

Sorasio, G., Marti, M., Fonseca, R., \& Silva, L. O. 2006, Phys. Rev. Lett., 96, 045005

Stasiewicz, K., \& Ekeberg, J. 2008, Nonl. Proc. Geophys., 15, 681

Tanimori, T., Hayami, Y., Kamei, S., et al. 1998, ApJ, 497, L25

Tokar, R. L., \& Gary, S. P. 1985, Phys. Fluids, 28, 106

Treumann, R. A., \& Baumjohann, W. 1997, Basic Space Plasma Physics (London: Imperial College Press)

Tzoufras, M., Ren, C., Tsung, F. S., et al. 2006, Phys. Rev. Lett., 96, 105002

Uchiyama, Y., Aharonian, F. A., Tanaka, T., Takahashi, T., \& Maeda, Y. 2007, Nature, 449, 576

Umeda, T., Yamao, M., \& Yamazaki, R. 2009, ApJ, 695, 574

Volk, H. J., Berezhko, E. G., \& Ksenofontov, L. T. 2005, A\&A, 444, 893

Winske, D., \& Leroy, M. M. 1984, J. Geophys. Res., 89, 2673

Zindo, A., Ohsawa, Y., Bessho, N., \& Sydora, R. 2005, Phys. Plasmas, 12, 052321 